\title{
Reduced Activity of AMP-Activated Protein Kinase Protects against Genetic Models of Motor Neuron Disease
}

\author{
M. A. Lim, ${ }^{1,2}$ M. A. Selak, ${ }^{4}$ Z. Xiang, ${ }^{5}$ D. Krainc, ${ }^{5}$ R. L. Neve, ${ }^{6}$ B. C. Kraemer, ${ }^{7}$ J. L. Watts, ${ }^{8}$ and R. G. Kalb ${ }^{1,2,3}$ \\ ${ }^{1}$ Department of Pediatrics, Division of Neurology, Abramson Research Center, Children's Hospital of Philadelphia, Philadelphia, Pennsylvania 19104, \\ ${ }^{2}$ Neuroscience Graduate Group and ${ }^{3}$ Department of Neurology, University of Pennsylvania, Philadelphia, Pennsylvania 19104, ${ }^{4}$ Department of Neurology, \\ Uniformed Services University of the Health Sciences, Bethesda, Maryland 20814, ${ }^{5}$ MassGeneral Institute for Neurodegenerative Disease, Charlestown, \\ Massachusetts 02129, ${ }^{6}$ Department of Brain and Cognitive Sciences, Massachusetts Institute of Technology, Cambridge, Massachusetts 02139, ${ }^{7} \mathrm{Geriatrics}$ \\ Research Foundation and Clinical Center, Veterans Affairs Puget Sound Health Care System, Seattle, Washington 98108, and ${ }^{8}$ School of Molecular \\ Biosciences, Washington State University, Pullman, Washington 99164
}

A growing body of research indicates that amyotrophic lateral sclerosis (ALS) patients and mouse models of ALS exhibit metabolic dysfunction. A subpopulation of ALS patients possesses higher levels of resting energy expenditure and lower fat-free mass compared to healthy controls. Similarly, two mutant copper zinc superoxide dismutase 1 (mSOD1) mouse models of familial ALS possess a hypermetabolic phenotype. The pathophysiological relevance of the bioenergetic defects observed in ALS remains largely elusive. AMPactivated protein kinase (AMPK) is a key sensor of cellular energy status and thus might be activated in various models of ALS. Here, we report that AMPK activity is increased in spinal cord cultures expressing mSOD1, as well as in spinal cord lysates from mSOD1 mice. Reducing AMPK activity either pharmacologically or genetically prevents mSOD1-induced motor neuron death in vitro. To investigate the role of AMPK in vivo, we used Caenorhabditis elegans models of motor neuron disease. C. elegans engineered to express human mSOD1 (G85R) in neurons develops locomotor dysfunction and severe fecundity defects when compared to transgenic worms expressing human wild-type SOD1. Genetic reduction of $a a k-2$, the ortholog of the AMPK $\alpha 2$ catalytic subunit in nematodes, improved locomotor behavior and fecundity in G85R animals. Similar observations were made with nematodes engineered to express mutant tat-activating regulatory (TAR) DNA-binding protein of $43 \mathrm{kDa}$ molecular weight. Altogether, these data suggest that bioenergetic abnormalities are likely to be pathophysiologically relevant to motor neuron disease.

\section{Introduction}

In amyotrophic lateral sclerosis (ALS), degeneration of both upper and lower motor neurons leads to progressive weakness and ultimately death. Contributing pathogenic processes include glu-

\footnotetext{
Received Dec. 15, 2010; revised Nov. 21, 2011; accepted Nov. 27, 2011.

Author contributions: M.A.L., M.A.S., D.K., B.C.K., J.L.W., and R.G.K. designed research; M.A.L., M.A.S., Z.X., R.L.N., B.C.K., J.L.W., and R.G.K. performed research; R.L.N. contributed unpublished reagents/analytic tools; M.A.L., Z.X., D.K., and R.G.K. analyzed data; M.A.L., M.A.S., and R.G.K. wrote the paper.

This work was supported by National Institutes of Health Grants F31NS06816502 (M.A.L.), R21NS060754, and R01NS052325 (R.G.K.), the Department of Veterans Affairs (B.C.K.), and the ALS Association. We thank the generosity of Dr. Morris Birnbaum (University of Pennsylvania, Philadelphia, PA) for the AMPK wild-type and dominant negative plasmids; Dr. David Borchelt (University of Florida, Gainesville, FL) for the wild-type and mutant SOD1 plasmids; Drs. Arthur Horwich (Yale University, New Haven, CT) and Jiou Wang (Johns Hopkins University, Baltimore, MD) for the WT SOD1, G85R mSOD1, and G85R(line 18) worm strains; Dr. Hyeon-Sook Koo (Yonsei University, Seoul, South Korea) for the AAK2::GFP translational reporter strain; Drs. Martin Chalfie, Irini Topalidou, and Xiaoyin Chen (Columbia University, New York, NY) for the sid-1 mutant strains; Dr. Yishi Jin (University of California at San Diego, La Jolla, CA) for the CZ1200 strain; Dr. Miriam Goodman (Stanford University, Palo Alto, CA) for sharing the Parallel Worm Tracker program; and Drs. Gary Ruvkun (Harvard University, Cambridge, MA) and Todd Lamitina (University of Pennsylvania, Philadelphia, PA) for sharing RNAi clones. We acknowledge the Gene Knockout Consortium at Oklahoma Medical Research Center, which provided several of the C. elegans knock-out strains used in our experiments. We also acknowledge Jelena Mojsilovic-Petrovic, Heather Pratt, Peter Krumbhaar, and Daniel Helbig for their excellent technical assistance. Finally, we thank the C. elegans laboratories at the University of Pennsylvania (Christopher Fang-Yen, Todd Lamitina, John Murray, David Raizen, and Meera Sundaram) for their advice and suggestions. The authors declare no competing financial interests.

Correspondence should be addressed to Robert G. Kalb at the above address. E-mail: kalb@email.chop.edu. DOI:10.1523/JNEUROSCI.6554-10.2012

Copyright $\odot 2012$ the authors $\quad 0270-6474 / 12 / 321123-19 \$ 15.00 / 0$
}

tamate excitotoxicity, protein misfolding, inflammation, and oxidative stress (Reviewed in Rothstein, 2009). Intriguingly, there is also evidence for defective energy homeostasis in patients and mouse models of ALS. A subpopulation of sporadic (sALS) and familial ALS (fALS) patients possesses higher levels of resting energy expenditure $(\sim 16-18 \%$ increase $)$ as measured by indirect calorimetry and lower fat-free mass compared to healthy controls (Kasarskis et al., 1996; Desport et al., 2005; Dupuis et al., 2008, 2011; Bouteloup et al., 2009; Funalot et al., 2009). Clinically, hypermetabolism is defined as the ratio of measured resting energy expenditure ( $\mathrm{mREE}$ ) and calculated resting energy expenditure (cREE), or mREE/cREE $\geq 1.1$ ( $\geq 10 \%$ increase). Hypermetabolism in ALS patients cannot be ascribed to changes in forced vital capacity, muscle fasciculations, fever, hyperthyroidism, or infection (Desport et al., 2001). Similarly, transgenic mouse models overexpressing mutant copper zinc superoxide dismutase 1 (mSOD1) (G86R and G93A mice) show increased energy expenditure by indirect calorimetry, increased lipid and glucose clearance in skeletal muscle, and hypolipidemia (Dupuis et al., 2004; Fergani et al., 2007; Kim et al., 2011) (Lim and Kalb, unpublished observations). Metabolic defects have also been reported previously in another potential model of motor neuron disease. Mice with conditional postnatal ablation of tat-activating regulatory (TAR) DNA-binding protein of $43 \mathrm{kDa}$ molecular weight (TDP-43) develop rapid weight loss and increased fatty 
acid oxidation, and experience early death (Chiang et al., 2010). At the cellular level, mitochondria from spinal cord and muscle of mSOD1 and TDP-43 mice possess both morphological and physiological abnormalities (Bendotti et al., 2001; Mattiazzi et al., 2002; Cassina et al., 2008; Pedrini et al., 2010; Shan et al., 2010; Xu et al., 2010; Zhou et al., 2010; Braun et al., 2011). Compromised mitochondrial function may contribute to systemic metabolic defects in the organismal level.

One-way cells sense a mismatch between energy supply and demand is through AMPK. A master sensor and regulator of energy balance, AMPK is a heterotrimeric protein, comprised of catalytic $\alpha$ subunits $(\alpha 1, \alpha 2)$, and $\beta$ and $\gamma$ regulatory subunits $(\beta 1, \beta 2, \gamma 1, \gamma 2, \gamma 3)$ (Hardie, 2007). When ATP supply does not keep pace with demand, ATP/AMP ratios fall, leading to the activation of AMPK. It is activated by events that either compromise cellular ATP production (i.e., oxidative stress, hypoxia, nutrient deprivation) or increase ATP consumption (exercise) (Kahn et al., 2005; Long and Zierath, 2006; Hardie, 2007). AMPK activation results in increased fatty acid oxidation and glucose uptake, as well as decreased protein translation, glycogen, and cholesterol synthesis.

AMPK's strategic position at the crossroads of multiple metabolic pathways led us to wonder whether AMPK participated in the metabolic dysfunction associated with neurodegeneration. In this report, we confirm the existence of metabolic defects in mSOD1-induced motor neuron disease and provide evidence that these defects are associated with activation of AMPK. Finally, we present in vitro and in vivo evidence that activated AMPK adversely affects not only mSOD1-induced, but also mutant TDP-43-induced motor neuron disease.

\section{Materials and Methods}

Mixed spinal cord neuron cultures. Mixed spinal cord neuron cultures were prepared as described previously (Jeong et al., 2006; MojsilovicPetrovic et al., 2006). Briefly, an astrocyte feeder layer was prepared from the cortex of newborn Sprague Dawley rat pups [postnatal day 2 (P2)] and grown to $\sim 80 \%$ confluency. Subsequently, dissociated embryonic day 15 (E15) spinal cord neurons were added. One to $2 \mathrm{~d}$ later, AraC (5 mM) (catalog \#C6645; Sigma) was added for $24 \mathrm{~h}$ to arrest astrocyte proliferation. Cultures were maintained in glia-conditioned medium supplemented with the following trophic factors $(1.0 \mathrm{ng} / \mathrm{ml}$ each): human neurotrophin-3, human neurotrophin-4, human brain-derived neurotrophic factor, human cardiotrophin-1, human glial-derived neurotrophic factor, and rat ciliary neurotrophic factor (Alomone Labs). Half of the culture medium was changed on a biweekly basis.

Recombinant herpes simplex virus constructs. Wild-type (WT) and mutant SOD1, wild-type AMPK $\alpha 2$, and dominant-negative AMPK $\alpha 2$ (dnAMPK; K45R), as well as Lac-Z cDNAs, were cloned into the PrpUC amplicon plasmid to generate recombinant herpes simplex virus (HSV) as described previously (Neve et al., 1997). The titer of virus used in these experiments was $\sim 3-5 \times 10^{7}$ plaque-forming units $/ \mathrm{ml}$.

Mouse care. Male heterozygote G93A mSOD1 mice on the congenic C57BL/6 background (Strain 004435; Jackson Laboratory) were backcrossed with C57BL/6 mice. Nontransgenic (non-Tg) littermates served as control mice. Both male and female G93A mSOD1 mice and control littermates were used for experimentation. Mice were fed a standard diet (Purina 5010) and were housed on a $12 \mathrm{~h}$ light/dark cycle. Genotypes were determined by PCR using tail snip DNA. Primers are listed in Table 1. All animals were treated in strict accordance with the National Institutes of Health Guide for the Care and Use of Laboratory Animals and were approved by the Children's Hospital of Philadelphia Institutional Animal Care and Use Committee.

Reverse-transcribed PCR and quantitative real-time PCR. Mixed spinal cord cultures and mouse tissues were lysed or homogenized in Trizol (Invitrogen) to extract RNA. Synchronized young adult Caenorhabditis elegans were lysed in Trizol, and RNA was purified using the RNeasy
Table 1. List of primers

\begin{tabular}{|c|c|}
\hline Gene & Primers ( $5^{\prime}$ to $\left.3^{\prime}\right)$ \\
\hline G93A mS0D1 (genomic DNA) & $\begin{array}{l}\text { cgcgactaacaatcaaagtga } \\
\text { catgagccctaatccatctga } \\
\text { gtaggtggaaattctagcatcatcc } \\
\text { ctaggccacagaattgaaagatct }\end{array}$ \\
\hline aak-2 (genomic DNA) & $\begin{array}{l}\text { atgtcgttggaaagattcgc } \\
\text { caatgctgaggtgacttcctcttcg }\end{array}$ \\
\hline sid-1 (genomic DNA) & $\begin{array}{l}\text { agctctgtacttgtattcg } \\
\text { gcacagttatcagatttg }\end{array}$ \\
\hline tbc-11 (genomic DNA) & $\begin{array}{l}\text { Outer } \\
\text { gatcatctcgtccgactctatg } \\
\text { agacacgtccacgaagcttatc } \\
\text { Inner } \\
\text { gcggcgtaaagttcaatctc } \\
\text { atcgctatccgattccagtg }\end{array}$ \\
\hline AMPK $\alpha 1$ & $\begin{array}{l}\text { atccgcagagagatccagaa } \\
\text { cgtcgactctcctttcgtc }\end{array}$ \\
\hline AMPK $\alpha 2$ & $\begin{array}{l}\text { agctcgcagtggcttatcat } \\
\text { ggggctgtctgctatgagag }\end{array}$ \\
\hline AMPK $\beta 1$ & $\begin{array}{l}\text { aggctccagagaaggaggag } \\
\text { actggccatccacaaagaac }\end{array}$ \\
\hline AMPK $\beta 2$ & $\begin{array}{l}\text { gcagcaggatttggatgatt } \\
\text { ggatcatgaacccactgtcc }\end{array}$ \\
\hline AMPK $\gamma 1$ & $\begin{array}{l}\text { ggagtcaggcaacaccttgt } \\
\text { atgcccagagccacatagac }\end{array}$ \\
\hline AMPK $\gamma 2$ & $\begin{array}{l}\text { tggagaccattgtggacaga } \\
\text { tgtgagggcgtctacacttg }\end{array}$ \\
\hline AMPK $\gamma 3$ & $\begin{array}{l}\text { ccacgagagectaggtgaag } \\
\text { ttccaagatcctttcgttgg }\end{array}$ \\
\hline GAPDH & $\begin{array}{l}\text { acccagaagactgtggatgg } \\
\text { cacattgggggtaggaacac }\end{array}$ \\
\hline $\mathrm{PGC1}-\alpha$ & $\begin{array}{l}\text { ttgcccagatcttcctgaacttg } \\
\text { caaatgagggcaatccgtcttca }\end{array}$ \\
\hline GPx1 & $\begin{array}{l}\text { gacctcgtggactggtggtg } \\
\text { gtcactgggtgttggcaagg }\end{array}$ \\
\hline Sod2 & $\begin{array}{l}\text { ggagagcagcggtcgtgtaa } \\
\text { cggtggcgttgagattgttc }\end{array}$ \\
\hline $\begin{array}{l}t b c-11 \\
c d c-42\end{array}$ & $\begin{array}{l}\text { tacttccgtgtgtccttcc } \\
\text { ccatcctcaacacagcgtct } \\
\text { ctgctggacaggaagattacg } \\
\text { ctcggacattctcgaatgaag }\end{array}$ \\
\hline
\end{tabular}

Primers used for genotyping by PCR of aak-2(ok524), tbc-11(ok2576), sid-1(pk3321), G85R;aak-2, G85R;tbc-11, G85R;aak-2;tbc-11, and G85R;;id-1 mutant worms, and G93A mSOD1 mice are shown. Primers were utilized to analyze expression of AMPK subunits, PGC- $1 \alpha$ and its downstream targets, as well as TBC1D1 and tbc- -11 mRNA in mixed spinal cord cultures, mouse tissues, and whole worm RNA lysates.

Micro Kit (Qiagen). All samples were checked for RNA concentration and purity and reverse transcribed to cDNA using the Superscript II Reverse Transcriptase kit (Invitrogen). For peroxisome proliferator activated receptor $\gamma$ coactivator-1 $\alpha$ (PGC- $1 \alpha)$, glutathione peroxidase 1 (Gpx1), iron/manganese superoxide dismutase 2 (Sod2), and glyceraldehyde 3-phosphate dehydrogenase (GAPDH), quantitative real-time PCR (QPCR) was performed on an iCycler Real-Time PCR Detection System (Bio-Rad) using SYBR green PCR mix (Invitrogen). For other samples, QPCR was performed using a StepOnePlus Real-time PCR system (Applied Biosystems) using TaqMan Gene Expression Master Mix (Applied Biosystems) or Power SYBR Green PCR Master Mix (Invitrogen). Two to three independent experiments with three to five samples each were performed for mixed spinal cord culture and C. elegans samples. For QPCR analyses, all data were normalized to appropriate housekeeping genes (GAPDH, 18sRNA, or $c d c-42$ ) and the comparative Ct (delta delta $\mathrm{Ct}$ ) method was used. TaqMan gene expression assays (Applied Biosystems) were used to probe for TBC1D1 (assay ID, Rn01413271_m1) and 18sRNA (assay ID, Hs99999901_s1). Other primers used are listed in Table 1.

Western blot analysis. Mixed spinal cord cultures were lysed and mouse tissues were homogenized on ice with lysis buffer (210 mM mannitol, 70 
mm sucrose, 10 mm HEPES, pH 7.2, and 1 mM EGTA supplemented fresh with $0.5 \% \mathrm{w} / \mathrm{v}$ fatty-acid free bovine serum albumin (BSA) and protease and phosphatase inhibitors). Lysates were sonicated (particulate matter was removed by centrifugation), and boiled in $1 \times$ SDS protein loading buffer and $1 \% \beta$-mercaptoetanol (BME). To prepare worm lysates, worms were washed off agar plates with worm lysis buffer $(50 \mathrm{~mm} \mathrm{KCl}, 10$ mu Tris-Cl, $\mathrm{pH}$ 8.3, $2.5 \mathrm{~mm} \mathrm{MgCl}_{2}, 0.45 \%$ Tween-20, $0.45 \% \mathrm{NP}-40$, and $0.01 \%$ gelatin, supplemented fresh with protease and phosphatase inhibitors). To wash the bacteria off the worms, samples were centrifuged once and resuspended in lysis buffer. Samples were sonicated and centrifuged at $99,000 \times g$. The supernatant was boiled in $1 \times$ SDS protein loading buffer and $1 \%$ BME and saved for probing of soluble proteins. The pellet was resuspended in lysis buffer and centrifuged again at $99,000 \times g$. The remaining pellet was boiled in SDS and 1\% BME and saved for probing of insoluble proteins.

All samples were subjected to SDS-PAGE, transferred to nitrocellulose membrane (Bio-Rad Laboratories), and blocked (5\% w/v of nonfat milk or BSA in $1 \mathrm{~mm}$ Tris-HCl, pH 7.5, and 0.1\% Tween). Membranes were incubated in primary antibody overnight, washed, and incubated with secondary fluorescent antibodies (infrared 800 or 680 anti-mouse IgG or anti-rabbit IgG; LI-COR Biosciences). Western blots were visualized and quantified using the LI-COR Odyssey Infrared Imaging System, and protein bands never saturated detectors. The following antibodies from Cell Signaling Technology were used: anti-AMPK (catalog \#2532), antiphospo-AMPK (pAMPK; catalog \#2535), anti-acetyl-coA carboxylase (ACC; catalog \#3676), anti-phospho-ACC (pACC; catalog \#3661), antifactor 4E binding protein 1 (4EBP1; catalog \#9452), anti-phospho4EBP1 (p4EBP1; catalog \#9451), anti-p70 S6 Kinase (p70 S6 Kinase; catalog \#9202), anti-phospho-p70 S6 Kinase (pp70 S6 Kinase; catalog \# 9205), and anti-human SOD1 (catalog \#2770). Anti-actin antibody (catalog \#A206) was from Sigma. Aliquots of samples were saved for protein concentration assays.

Oxygen consumption assays. Oxygen consumption was measured polarographically in intact cells using a magnetically stirred, thermostatically regulated $\left(37^{\circ} \mathrm{C}\right)$ Mitocell Clark-type electrode system using a chamber volume of $150 \mu \mathrm{l}$ (Model MT200; Strathkelvin Instruments). For these studies, mixed spinal cord cultures were resuspended by trypsinization, neutralized with culture media, washed, and then resuspended in fresh culture medium without serum. An aliquot of each sample was saved for protein quantification. A total of $150 \mu \mathrm{l}$ of cell suspension was loaded into the chamber. Sequential measurements were made of the basal respiratory rate following addition of $0.5 \mu \mathrm{g} / \mathrm{ml}$ of the mitochondrial ATP synthase inhibitor oligomycin and maximal respiration stimulated by addition of the uncoupler carbonyl cyanide $m$-chlorophenylhydrazone (CCCP; $30 \mu \mathrm{M})$. ATP turnover was calculated by subtracting oligomycin-insensitive respiration from the basal respiration rate to yield oligomycin-sensitive oxygen consumption, and proton leak was determined from oligomycin-insensitive respiration. All values were normalized to protein content and are reported as nanoatoms Oxygen per minute per milligram protein.

We also measured mitochondrial respiration in mSOD1 mouse spinal cord homogenates. P40 and P90 G93A mSOD1 and non-Tg control mouse spinal cords were homogenized with 10 strokes of a dounce homogenizer using a tight pestle to prepare $10 \% \mathrm{w} / \mathrm{v}$ homogenates in icecold mitochondrial buffer $(210 \mathrm{~mm}$ mannitol, $70 \mathrm{~mm}$ sucrose, $10 \mathrm{~mm}$ HEPES, pH 7.2, and 1 mM EGTA) freshly supplemented with $0.5 \% \mathrm{w} / \mathrm{v}$ of fatty-acid free BSA and protease and phosphatase inhibitors. A total of $150 \mu \mathrm{l}$ of whole tissue homogenate was loaded into the electrode chamber. Complex I-dependent respiration was measured using $10 \mathrm{~mm}$ glutamate plus $2 \mathrm{~mm}$ malate followed by addition of ADP $(0.2 \mathrm{~mm})$ to stimulate State 3 respiration. The respiratory control ratio (RCR; State $3 /$ State 2) was calculated as the ratio of the rate of oxygen consumption after ADP addition (State 3 ) to that obtained before addition of ADP (State 2). Sensitivity to oligomycin and CCCP were also tested. All reagents were from Sigma and were of the highest grade possible. The results of this method yielded similar respiration rates compared to isolated mitochondrial preparations.

In vitro $m S O D 1$ toxicity assays. After $14 \mathrm{~d}$ in vitro (DIV), mixed spinal cultures were infected with either HSV-WT SOD1 or HSV-mSOD1
(G37R). Viral stock $(2 \mu \mathrm{l})$ was added to $1 \mathrm{ml}$ of culture media $24 \mathrm{~h}$ or more before the next manipulation. When more than one recombinant HSV vector was used, $1.5 \mu$ lof each viral stock was added, yielding a total of $3 \mu \mathrm{l}$ of viral stocks to $1 \mathrm{ml}$ of media. Previous work has shown that both transgenes are expressed in $>95 \%$ of neurons in a nontoxic manner under these circumstances (Mojsilovic-Petrovic et al., 2009). To quantify motor neurons, immunostained cells were counted in four to five randomly selected fields in each coverslip. In each experiment, three or more coverslips were used per condition, and the results presented are from at least three independent experiments. Experimenter was blind to all groups.

For the pharmacology experiment, cultures were treated with either Compound C (CC; $5 \mu \mathrm{M}$; catalog \#171260; Calbiochem), aminoimidazole carboxamide ribonucleotide (AICAR) (10 mM; catalog \#123040; Calbiochem), or vehicle (DMSO) every $2 \mathrm{~d}$ for $7 \mathrm{~d}$. On the seventh day, cultures were fixed in $4 \%$ paraformaldehyde. Motor neurons were identified in mixed culture by immunostaining with anti-SMI-32 antibody (catalog \#SMI-32R; Covance), which specifically stains for nonphosphorylated neurofilaments. Only labeled cells with cell body diameter $25 \mu \mathrm{m}$ or greater were counted. This method has been validated previously as a means of recognizing motor neurons (Mojsilovic-Petrovic et al., 2006, their Fig. 1). All graphs are presented as the percentage of SMI-32positive neurons in MSOD1-infected compared to WT SOD1-infected cultures treated with the same drugs or constructs.

C. elegans. The following C. elegans strains were obtained from the $C$. elegans Genetic Center: the N2 Bristol strain, aak-2 null (ok524; strain RB754), sid-1 ( $p k 3321$; strain NL3321), and tbc-11 null (ok2576; strain RB1959). The mutant lines Psnb-1::WT SOD1-YFP, Psnb-1::G85R SOD1-YFP, and Psnb-1::G85R SOD1 (line 18)-YFP were generous gifts from Arthur Horwich (Yale University, New Haven, CT) and Jiou Wang (Johns Hopkins University, Baltimore, MD), and were created as described previously (Wang et al., 2009). The mutant lines Psnb-1::TDP-43(WT) and Psnb-1::TDP-43(M337V) were generated as described previously (Liachko et al., 2010). Compound mutant strains, which were null for $a a k-2$ and $t b c-11$, were confirmed by fluorescence and PCR using primers for $a a k-2$ and $t b c-11$ (see Table 1 ). The following strains were used to generate $G 85 R$ worms expressing sid-1 in the nervous system: sid-1(pk3321) and uIS69 [pCFJ90 (Pmyo-2mCherry); Punc119sid-1];sid-1(pk3321). The latter was a generous gift from Martin Chalfie (Columbia University, New York, NY) and was generated as described previously (Calixto et al., 2010). Triple-mutant worms, Psnb-1::G85R-YFP;[pCFJ90 (Pmyo-2mCherry); Punc-119sid-1];sid-1(pk3321), were confirmed by yellow fluorescent protein (YFP) fluorescence and PCR primers for sid-1 (see Table 1). A G-to-A mutation in the pk3321 allele of sid- 1 creates an ApoI restriction digest site, and this was used for genotyping. For the neurodegenerative assays, TDP-43(M337V) and TDP-43(M337V); aak-2(ok424) worms were crossed into the background strain CZ1200 (a generous gift from Yishi Jin, University of California at San Diego, La Jolla, CA) as described previously. This strain contains an integrated Punc-25::GFP transgene expressed in GABAergic neurons that clearly marks the cell bodies and axons of ventral cord ventral D (VD)- and dorsal D (DD)-type inhibitory motor neurons. All animals were grown on Nematode Growth Media (NGM) plates $(\sim 10 \mathrm{ml}$ agar) seeded with Escherichia coli OP50. All animals were maintained at $20^{\circ} \mathrm{C}$.

Locomotor assays. C. elegans locomotor behavior was tested in two different assays: (1) a crawling assay performed on agar plated with OP50 bacteria and (2) a swimming assay performed on a drop of M9 buffer on the surface of an agar plate. A total of 15 to 25 worms were allowed to lay eggs for $4 \mathrm{~h}$. Once larvae reached the L4 stage, locomotor behavior was blindly recorded on a videocamera attached to a Zeiss Stemi SV11 dissecting scope and tracked using the Parallel Worm Tracker program (http://wormsense.stanford.edu/tracker; Miriam Goodman, Stanford University, Palo Alto, CA). Behavior was also recorded at an adult stage (24 $\mathrm{h}$ after L4). On average, five replicates containing 7-10 animals per group were tested, and at least two independent experiments were performed for each locomotor assay. To analyze crawling and swimming behavior, animal tracks and size were analyzed on MATLAB (MathWorks) to determine average speed (millimeters per second) and average speed normalized to size. 


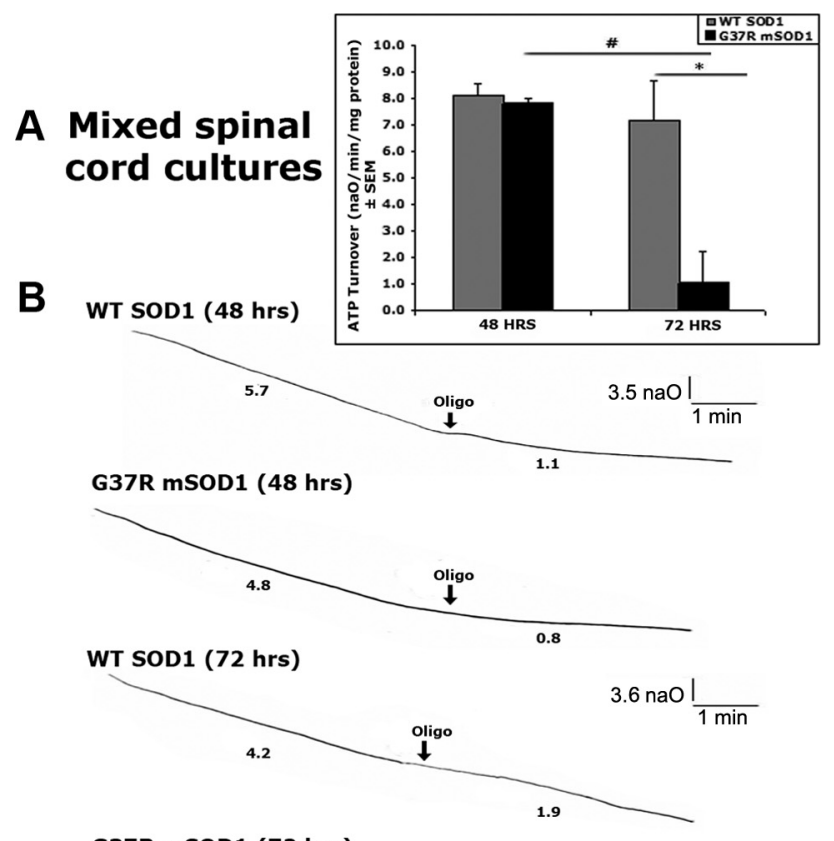

G37R mSOD1 (72 hrs)

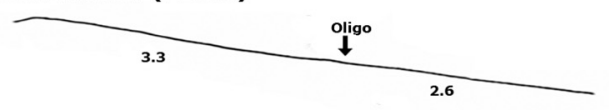

C Mouse spinal cord

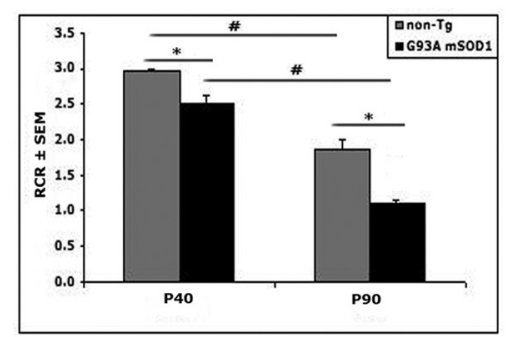

D
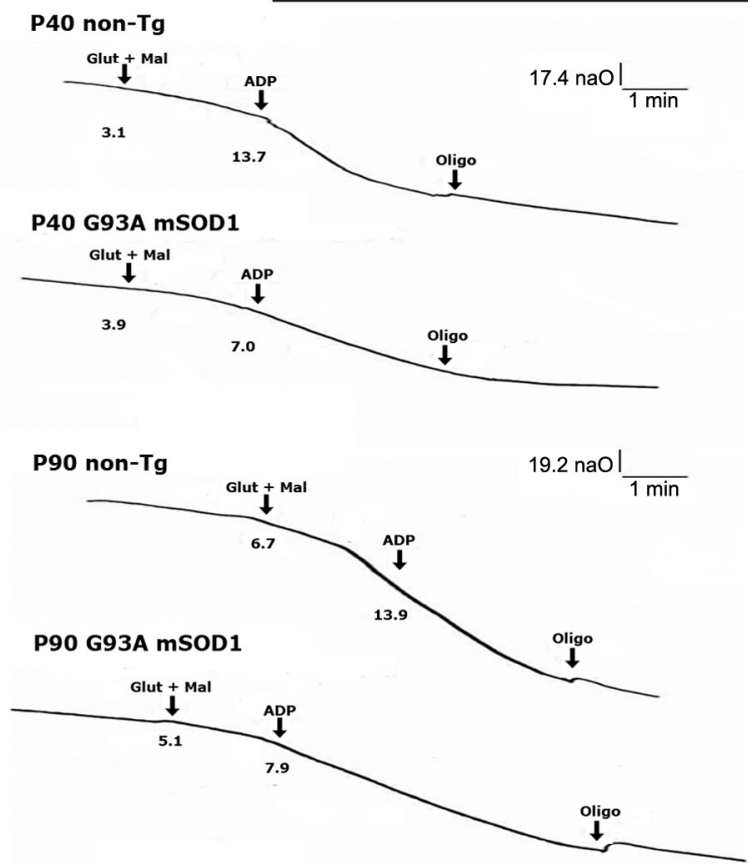

Figure 1. Bioenergetic abnormalities are present in MSOD1 models of motor neuron disease. A, ATP turnover (respiration supporting ATP synthesis) in whole-cell extracts from mixed spinal cord cultures infected for 48 or $72 \mathrm{~h}$ with either HSV-WTSOD1 or G37R mSOD1 virus. ${ }^{*} p \leq$ 0.01 , significantly different from $48 \mathrm{~h}$ time point; ${ }^{*} p<0.01$, significantly different from WT
RNAi knockdown in C. elegans. Each RNA interference (RNAi) colony was grown overnight in Luria broth containing ampicillin $(50 \mu \mathrm{g} / \mathrm{ml})$, and $200 \mu \mathrm{l}$ was seeded on NGM plates containing isopropylthiogalactoside $(1 \mathrm{~mm})$ to induce dsRNA expression. RNAi clones for aak-2 and tbc-11 were generated as described previously (Fraser et al., 2000) and were obtained from Gary Ruvkun (Harvard University, Cambridge, MA) and Todd Lamitina (University of Pennsylvania, Philadelphia, PA), respectively. For locomotor experiments, L4 animals were placed on RNAi plates overnight. On the next day, young adult animals were transferred to a fresh RNAi plate and allowed to lay eggs for another 4-6 h. Progeny was subsequently tested for locomotor activity.

Fecundity assays. Assays were performed as described previously by Soukas et al. (2009). Briefly, L4 animals were singled and transferred daily to fresh NGM plates. The total number of hatched embryos was counted $3 \mathrm{~d}$ later, and total brood size was determined by summing viable offspring across all days. The reproductive span was recorded from the day of first egg lay until the last day when laid eggs hatched into live offspring. Sterile worms, embryos that failed to hatch, and dead or unfertilized embryos were also recorded.

Neurodegeneration assays. Timed egg lays were arranged to produce synchronized populations at $48 \mathrm{~h}$ (L4) and $72 \mathrm{~h}$ (young adult). Live worms were placed on a $3 \%$ agarose pad containing $0.01 \%$ sodium azide to immobilize the worms. Worms were imaged under fluorescence microscopy and scored for number of GABAergic neurons, gaps in the ventral nerve cord, and gaps in the dorsal nerve cord.

Statistics. Statistics were analyzed using Statview 5.0 software (SAS Institute). One-way ANOVAs were used in comparisons between twogroup data and multivariate ANOVAs, including repeated-measures ANOVAs, were used in comparisons among three or more groups of data. Post hoc tests were performed to confirm significance. The threshold for significance was set at $p \leq 0.05$.

\section{Results}

Bioenergetic abnormalities are present in both in vitro and in vivo mSOD1 models of motor neuron disease

We began by asking whether bioenergetic defects can be detected in a mutant SOD1 (mSOD1) in vitro model of motor neuron disease. In previous work, we showed that expressing mSOD1, but not the wild-type human protein (WT SOD1), in mixed spinal cord cultures leads to time-dependent death of motor neurons beginning after $48 \mathrm{~h}$ (Mojsilovic-Petrovic et al., 2006). We measured mitochondrial respiration polarographically with a Clark-type oxygen electrode in intact cells from mixed spinal cord cultures expressing either HSV-WT SOD1 (WT SOD1) or HSV-G37R mSOD1 (mSOD1) for 48 and $72 \mathrm{~h}$. Since intact cells are not permeable to ADP and many mitochondrial substrates, analysis of their bioenergetic status cannot be performed in the same direct manner as isolated mitochondria or cell/tissue homogenates (Jekabsons and Nicholls, 2004). However, oligomycin, a mitochondrial ATP synthase inhibitor, is cell permeable and thus can be used to good advantage to examine the energetic status of intact cells.

The basal rate of oxygen consumption minus the rate following addition of oligomycin (ATP turnover, or "respiration supporting ATP synthesis") provides an estimate of oligomcyin-

$\leftarrow$

SOD1; $n=4-9$ dishes per group. $\boldsymbol{B}$, Representative polarographs of whole-cell extracts from HSV-WT SOD1 and HSV-G37R mSOD1 cultures infected for 48 and $72 \mathrm{~h}$. Numbers under the curves represent the slope of each segment of the trace in nanoatoms $0 x y$ gen per minute. $C, R C R$ of spinal cord homogenates from P40 and P90 mice. ${ }^{\#} p<0.0001$, significantly different from P40 time point; ${ }^{*} p<0.01$, significantly different from non-Tg controls; $n=4-8$ animals per group. $\boldsymbol{D}$, Representative polarographs in the presence of glutamate $(10 \mathrm{~mm})$ and malate $(2 \mathrm{~mm})$ in mouse spinal cord homogenates from P40 and P90 G93A mS0D1 mice and non- $\mathrm{Tg}$ control counterparts. Numbers under the curves represent the slope of each segment of the trace in nanoatoms 0 xygen per minute. Error bars represent \pm SEM. 
sensitive ATP turnover in the cell coupled to oxidative phosphorylation and thus provides an indication of the functional capability of mitochondria. In addition, oligomycin-insensitive respiration provides an indication of $\mathrm{H}^{+}$leak across the inner mitochondrial membrane, which decreases the efficiency of oxidative phosphorylation. CCCP-induced respiration provides a measure of maximum electron transport chain activity under uncoupled conditions.

Cultures infected with G37R mSOD1 virus showed a timedependent decrease in mitochondrial respiration $\left(F_{(1,11)}=9.05\right.$; $p \leq 0.01$ ) (Fig. $1 A, B)$. Furthermore, at $72 \mathrm{~h}$ after infection, mSOD1-infected cultures showed significantly decreased values for mitochondrial respiration compared to WT SOD1 counterparts $\left(F_{(1,17)}=9.32 ; p<0.01\right)$ (Fig. $\left.1 A, B\right)$. More specifically, ATP turnover in mSOD1-expressing neurons decreased by $>85 \%$ compared to WT SOD1-expressing neurons. Although basal rates of respiration showed a decreasing trend in mSOD1expressing neurons $(9.10 \pm 2.08 \mathrm{na} \mathrm{O} / \mathrm{min} / \mathrm{mg}$ in $\mathrm{mSOD} 1$ vs $12.44 \pm 1.17 \mathrm{na} \mathrm{O} / \mathrm{min} / \mathrm{mg}$ protein in WT SOD1), this was not statistically significant. There was also an increasing trend toward oligomycin-insensitive respiration in mSOD1- versus WT SOD1-expressing neurons $(8.10 \pm 1.40 \mathrm{na} \mathrm{O} / \mathrm{min} / \mathrm{mg}$ in $\mathrm{mSOD} 1$ vs $5.24 \pm 1.31 \mathrm{na} \mathrm{O} / \mathrm{min} / \mathrm{mg}$ protein in WT SOD1), which indicates increased $\mathrm{H}^{+}$leakiness of the inner mitochondrial membrane. There was neither a change in basal level nor oligomycin-insensitive respiration in mSOD1-infected cultures at $48 \mathrm{~h}$. In summary, data consistently reveal impaired mitochondrial respiration in mSOD1-expressing spinal cord neurons, possibly due to proton leak across the inner mitochondrial membrane.

We used a similar assay to measure respiration in a crude mitochondrial preparation from spinal cord of presymptomatic (P40) and symptomatic (P90) mSOD1 (G93A) mice. Mitochondrial respiration was measured in the presence of the complex I-linked substrate pair glutamate plus malate followed by addition of ADP, which stimulates oxygen consumption in wellcoupled mitochondria. The rate of oxygen consumption upon addition of ADP and after most ADP has been phosphorylated into ATP (State 3/State 4 ) yields the RCR. Because it was not always possible to measure a true State 4 , RCRs were calculated using State 2 (oxygen consumption upon addition of glutamate and malate and before addition of ADP) or oligomycininsensitive respiration. These values yielded similar results. The RCR reflects the degree of coupling between substrate oxidation and ADP phosphorylation and thus reports the functional integrity of mitochondria.

Addition of glutamate plus malate induced mitochondrial respiration in $\mathrm{P} 40$ and $\mathrm{P} 90 \mathrm{mSOD} 1$ mice and non-Tg control littermates to a similar extent (data not shown). There was an age-dependent decrease in mitochondrial respiration between P40 and P90 mouse spinal cord mitochondria in both non- $\mathrm{Tg}$ and mSOD1 mice $\left(F_{(1,19)}=116.30 ; p<0.0001\right)$ (Fig. $\left.1 C, D\right)$. However, ADP elicited a significantly smaller stimulation of oxygen consumption in mSOD1 mitochondria compared to controls, yielding a decreased RCR at both P40 and P90 (Fig. 1C,D). More specifically, at $\mathrm{P} 40$, the average RCR value in $\mathrm{mSOD} 1 \mathrm{mi}$ tochondria was reduced by $\sim 15 \%$ compared to control levels $(2.482 \pm 0.09$ compared to control littermates at $2.96 \pm 0.04)$ $\left(F_{(1,6)}=14.39 ; p<0.01\right)$. At P90, the average RCR in mSOD1 mitochondria was reduced by $\sim 40 \%$ compared to control levels $\left(1.1 \pm 0.04\right.$ compared to controls at $1.86 \pm 0.15 ; F_{(1,13)}=25.93$; $p<0.001)$. We also examined oligomycin sensitivity and obtained similar results when State 3/oligomycin-insensitive rates
Table 2. AMPK subunit expression in mixed spinal cord cultures and mouse tissues

\begin{tabular}{|c|c|c|c|}
\hline AMPK subunit & Mixed spinal cord culture & Spinal cord & Muscle \\
\hline \multicolumn{4}{|l|}{$\alpha$} \\
\hline$\alpha 1$ & + & + & \\
\hline$\alpha 2$ & + & + & \\
\hline \multicolumn{4}{|l|}{$\beta$} \\
\hline$\beta 1$ & + & + & \\
\hline$\beta 2$ & + & + & \\
\hline \multicolumn{4}{|l|}{$\gamma$} \\
\hline$\gamma 1$ & + & + & \\
\hline$\gamma 2$ & + & + & \\
\hline$\gamma 3$ & - & - & + \\
\hline
\end{tabular}

AMPK $\alpha 1, \alpha 2, \beta 1, \beta 2, \gamma 1, \gamma 2$, and $\gamma 3$ subunits are present in mouse spinal cord and mixed spinal cord cultures. $\gamma 3$ is not present in both samples, but is expressed in a positive control tissue from mouse (muscle). GAPDH was used as positive control and was expressed in all tissues (data not shown).

were used to calculate RCRs. No differences were observed in basal respiration rates before addition of glutamate plus malate (data not shown).

In addition to these mitochondrial abnormalities, P40 mSOD1 spinal cord mitochondria showed increased oligomycininsensitivity at $5.19 \pm 0.61 \mathrm{na} \mathrm{O} / \mathrm{min} / \mathrm{mg}$ protein compared to $1.37 \pm 0.77 \mathrm{na} \mathrm{O} / \mathrm{min} / \mathrm{mg}$ protein in non- $\mathrm{Tg}$ mice $\left(F_{(1,7)}=15.50\right.$; $p<0.006)$. There was a trend toward increased oligomycin insensitivity in P90 mSOD1 mice compared to non-Tg controls, but this was not significant. There was no difference in response to the uncoupler CCCP between $\mathrm{mSOD} 1$ mice and non- $\mathrm{Tg}$ counterparts at both P40 and P90 time points. In summary, these results demonstrate that bioenergetic abnormalities are present in both in vitro and in vivo mSOD1 models of motor neuron disease.

\section{AMPK $\alpha 1$ and $\alpha 2$ subunits are expressed in spinal cord cultures and spinal cord}

Considering the observed bioenergetic defects, we wondered if the energy sensor and regulator AMPK is activated in mSOD1 models. We began by investigating which AMPK subunits were expressed in our cultures and in rodent spinal cord because manipulation of specific subunits leads to distinct phenotypes (Viollet et al., 2003). Transcript expression of the AMPK $\alpha, \beta$, and $\gamma$ subunits, together with the housekeeping gene GAPDH, were therefore assayed by PCR analyses in mixed spinal cord cultures and mouse spinal cord cDNA (Table 2). Both $\alpha 1$ and $\alpha 2$ subunits, $\beta 1$ and $\beta 2$ subunits, as well as two of the three $\gamma$ subunits were expressed. The $\gamma 3$ subunit was present only in control cDNA from isolated mouse muscle (Table 2). These data are consistent with previous studies (Turnley et al., 1999).

\section{AMPK activity is increased in both in vitro and in vivo mSOD1 models of motor neuron disease}

Given that the AMPK catalytic subunits are present in our in vitro and in vivo mSOD1 models, we then asked whether the activity of AMPK and its downstream targets are altered as a result of observed defects in ATP production. We therefore assessed AMPK activity by immunoblotting for active AMPK (pAMPK at threonine 172), AMPK, and several downstream targets, such as ACC (pACC at serine 79 and ACC), factor 4E binding protein 1 (p4EBP1 at threonine 37/46 and 4EBP1), and p70 S6 kinase (pp70 S6 Kinase at threonine 389 and p70 S6 Kinase). ACC is a direct target of AMPK, and when phosphorylated, results in increased fatty acid oxidation. The proteins $4 \mathrm{EBP} 1$ and p70 S6 Kinase are downstream targets of the mammalian target of rapamycin (mTOR) pathway, which regulates protein transla- 
tion. AMPK activation leads to decreased activity of the mTOR pathway, which leads to decreased phosphorylation of 4EBP1 and p70 S6 Kinase and, consequently, reduced protein translation.

We prepared lysates from mixed spinal cord cultures infected with either HSV-WT SOD1 or HSV-G37R mSOD1. In mSOD1infected cultures, pAMPK expression was significantly increased compared to WT SOD1 counterparts, with no overall change in AMPK expression $\left(F_{(1,7)}=23.52 ; p<\right.$ 0.003; four independent experiments) (Fig. 2A,B). More specifically, the ratio of pAMPK/AMPK was increased by $\sim 50 \%$ in mSOD1-infected cultures. We also observed an approximate twofold increase in the ratio of pACC/ACC, which is consistent with increased AMPK activation $\left(F_{(1,14)}=7.14 ; p \leq 0.02\right.$ ) (Fig. $\left.2 A, B\right)$. Furthermore, the downstream targets of the mTOR pathway, 4EBP1 and p70 S6 Kinase, showed an $\sim 40 \%$ decrease in protein phosphorylation when normalized to overall protein levels, which is consistent with an activated AMPK state (Fig. 2A) (quantitative data not shown; two independent experiments). We next examined spinal cord lysates from G93A mSOD1 mice and control counterparts. At P40, there was no clear change in the expression patterns of pAMPK, AMPK, or its downstream target ACC (Fig. 2C,E). However, in P90 mSOD1 spinal cord, pAMPK expression increased, with no change in overall AMPKlevels, resulting in a 90\% increase in the pAMPK/AMPK ratio $\left(F_{(1,14)}=148.80 ; p \leq\right.$ 0.001) (Fig. 2. $D, E$ ). AMPKactivation was also associated with a $60 \%$ increase in the ratio of pACC/ACC compared with controls $\left(F_{(1,7)}=68.69 ; p \leq 0.0002\right)$ (Fig. $\left.2 D, E\right)$. An increased pAMPK/AMPK ratio was specific to the spinal cord, and observed in neither another nervous system tissue, such as the cerebellum, nor a peripheral tissue, liver.

Transcript levels of PGC-1 $\alpha$ and its downstream targets are not altered in spinal cord neurons and muscle of mSOD1 mice

AMPK regulates many transcriptional and translational targets that may play a role in metabolism, aging, and neurodegeneration. One such target of particular interest is PGC- $1 \alpha$, a transcriptional coactivator involved in mitochondrial biogenesis, antioxidant activity, and fiber-type switching (Puigserver and Spiegelman, 2003; Finck and Kelly, 2006). Furthermore, PGC- $1 \alpha$ has been shown to be regulated by AMPK (Jager et al., 2007; Irrcher et al., 2008). Since PGC- $1 \alpha$ dysregulation has been implicated in other neurodegenerative diseases, such as Huntington's disease and Parkinson's disease (Cui et al., 2006; Weydt et al., 2006, 2009; Chaturvedi et al., 2009; McConoughey et al., 2010; Hathorn et al., 2011; Shin et al., 2011), we wondered about its potential role in in vitro and in vivo mSOD1 models. We assessed the expression of PGC- $1 \alpha$ and its downstream targets, GPx1 and Sod2, enzymes involved in detoxifying free radicals. Compared to HSV-WT SOD1-infected cultures, mixed spinal
C

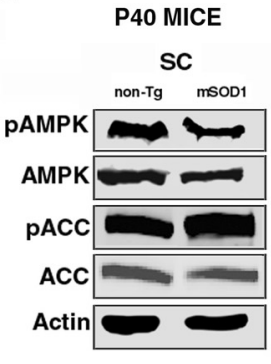

D
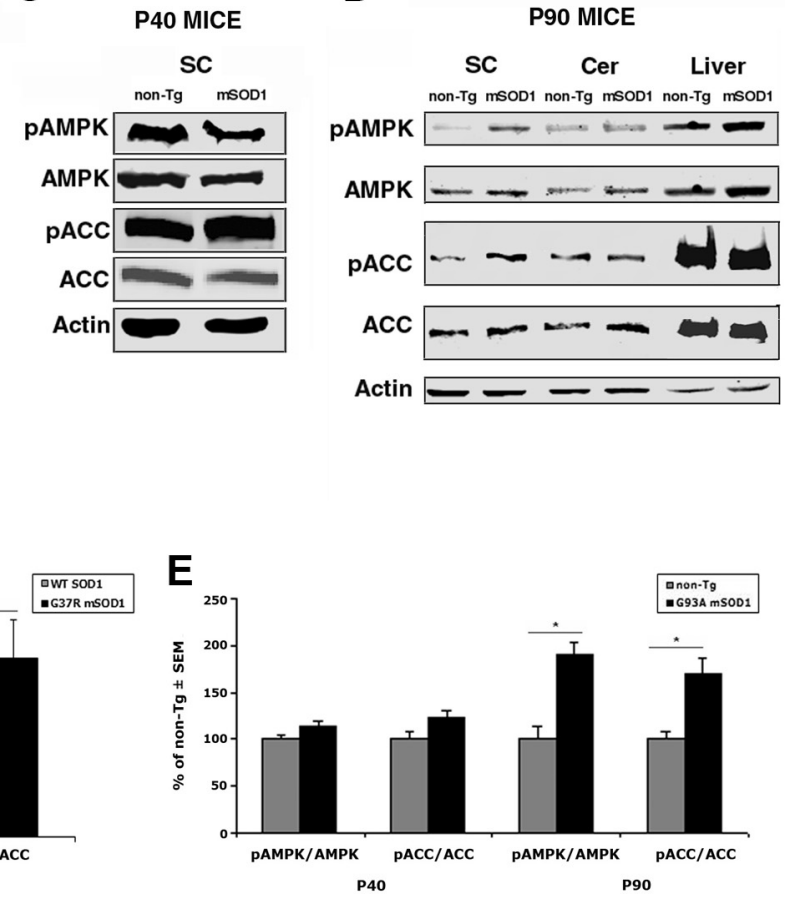

Figure 2. Increased AMPK activity in mSOD1 models of motor neuron disease. A, Mixed spinal cord cultures infected with either HSV-WT SOD1 or HSV-G37R mSOD1 for 48 h. pAMPK is significantly increased in mSOD1-infected compared to WT SOD1-infected , with no change in overall AMPK levels. The expression of the AMPK downstream target pACC is also increased, with no 列 ( between WT SOD1 and G37R mSOD1) from spinal cord of P40 and P90 mice. $n=\sim 5$ animals per group. For $\boldsymbol{B}$ and $\boldsymbol{E}$, data are presented as percentages compared to controls (either HSV-WT SOD1 or non-Tg controls). Error bars indicate \pm SEM.

cord cultures infected with HSV-G37R mSOD1 did not show any changes in the levels of PGC- $1 \alpha$ or its transcriptional target GPx1 (Sod2 was not detectable in our mixed spinal cord cultures) (Fig. $3 A$ ). Furthermore, no differences were observed in PGC- $1 \alpha$, GPx1, and Sod 2 in spinal cord and hindlimb muscle from P120 mice (Fig. $3 B, C)$.

\section{AMPK knockdown is neuroprotective in an in vitro mSOD1 model of motor neuron disease}

Is enhanced AMPK activity in MSOD1-expressing cultures beneficial or detrimental to neuronal health? We began by treating HSV-infected G37R mSOD1 mixed spinal cord cultures for $48 \mathrm{~h}$ with the AMPK antagonist CC $(5 \mu \mathrm{M})$, a competitive inhibitor of AMP binding sites, or vehicle (DMSO). To test drug efficacy, we performed immunoblots on cell lysates infected with WT or mSOD1. In both conditions, CC administration resulted in decreased abundance of pAMPK $\left(F_{(1,3)}=314.14 ; p \leq 0.003\right)$ and $\operatorname{pACC}\left(F_{(1,3)}=9411.19 ; p \leq 0.0001\right)$, with no change in the abundance of the nonphosphorylated species (Fig. $4 A, B$ ). The effect of Compound $\mathrm{C}$ was dose dependent (across 0, 5, 10 and $100 \mu \mathrm{M}$ dosages) (data not shown). A dose of $5 \mu \mathrm{M} \mathrm{CC}$ was then selected since it was the lowest dose with which a significant decrease in both pAMPK/AMPK and pACC/ACC levels were observed.

We next conducted $\mathrm{mSOD} 1$-induced toxicity assays in mixed spinal cord cultures infected with either HSV-WT SOD1 or G37R 
A
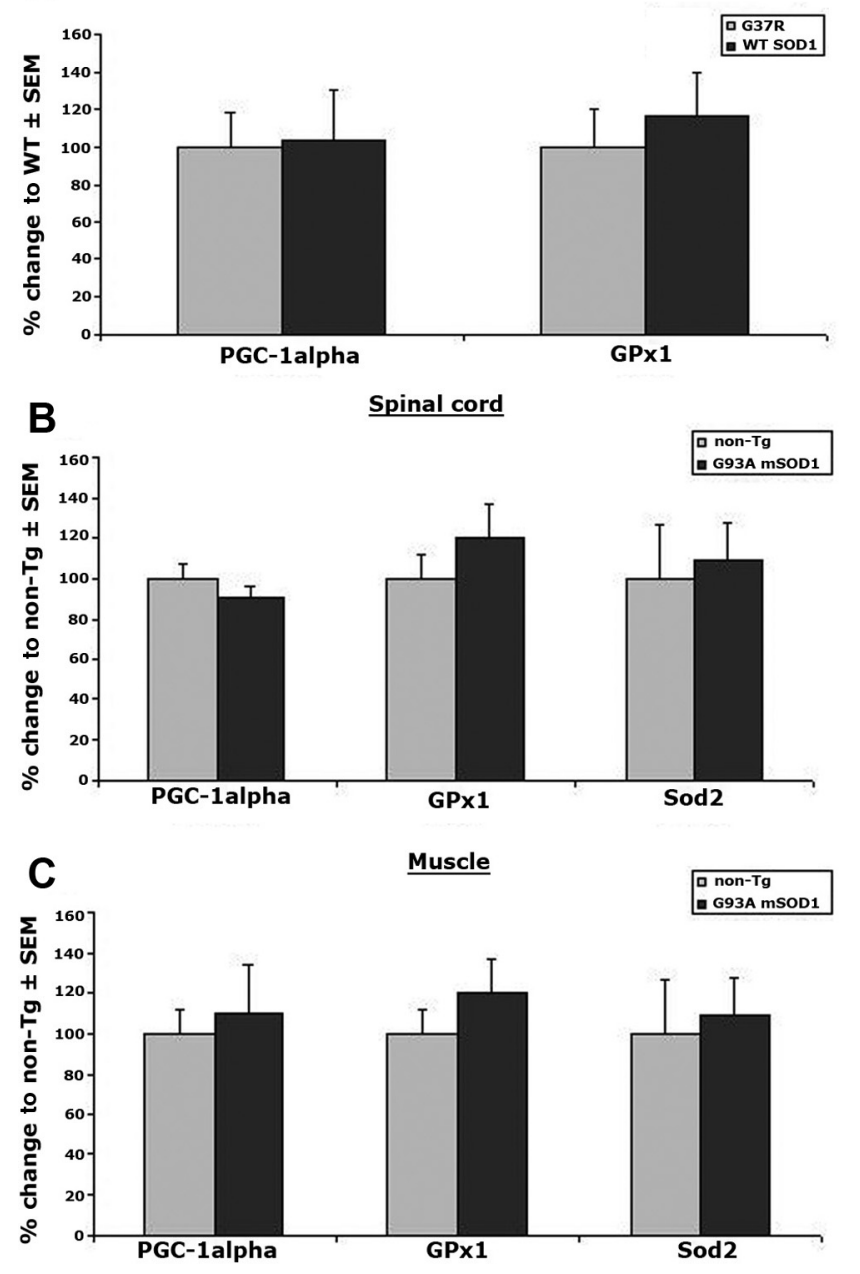

Figure 3. $\mathrm{PGC}-1 \alpha$ and downstream target transcript levels in in vitro and in vivo $\mathrm{mSOD} 1$ models of motor neuron disease. $A$, Mixed spinal cord cultures infected with HSV-G37R show no difference in PGC $-1 \alpha$ transcript levels and the levels of the antioxidant enzyme GPX1 compared to controls, HSV-WT SOD1 $(n=4-5$ dishes per group, three independent experiments). $\boldsymbol{B}$, Spinal cords from P120 G93A mice also reveal no differences in the expression levels of PGC $-1 \alpha$ and the antioxidant enzymes GPx1 and Sod2 compared to non-Tg controls. C, Hindlimb muscles from P120 G93A mS0D1 animals do not show a significant increase in mRNA levels of PGC-1 $1 \alpha$ and its downstream targets GPx1 and Sod2. For $\boldsymbol{B}$ and $\boldsymbol{C}, n=5-7$ animals per group. For $\boldsymbol{A}-\boldsymbol{C}$, all data are normalized to GAPDH and are presented as percentages compared to controls (either HSV-WT SOD1 or non-Tg controls). Error bars indicate \pm SEM.

mSOD1. Cultures were treated with either CC $(5 \mu \mathrm{M})$ or DMSO every $2 \mathrm{~d}$ for $7 \mathrm{~d}$ (three doses total). By ANOVA, significant groups were found $\left(F_{(3,88)}=7.33 ; p \leq 0.0002\right)$. Mixed cultures infected with mSOD1 and treated with DMSO demonstrated $~ 50 \%$ survival in SMI-32(+) neurons compared to WT SOD1-infected cultures treated with DMS0 ( $p \leq 0.002)$. This mSOD1-induced motor neuron death was completely blocked by CC administration $\left(F_{(1,4)}=\right.$ $18.88 ; p \leq 0.01$; three independent experiments) (Fig. $4 C$ ). We also tested the effects of the AMPK activator AICAR (10 mM) administration and found that it had no effect on mSOD1-infected cultures. Furthermore, mSOD1 cultures treated with AICAR showed significantly decreased motor neuron survival compared to CC-treated cultures $\left(F_{(1,46)}=85.77 ; p<0.001\right)$. There were no adverse effects of drug administered to cultures infected with WT SOD1 and treated with CC (data not shown).

In our second in vitro experiment, we used the same mixed culture conditions and toxicity assay to genetically manipulate
AMPK activity using a dominant negative construct of AMPK as described previously (Mu et al., 2001). HSV vectors were engineered to express WT AMPK or dnAMPK. To test efficacy of these constructs, we performed immunoblots on cell lysates infected with WT or mSOD1. In both conditions, coinfection with a dnAMPK construct resulted in decreased abundance of $\operatorname{pAMPK}\left(F_{(1,3)}=38.21 ; p<0.03\right)$ and $\operatorname{pACC}\left(F_{(1,6)}=5.69 ; p \leq\right.$ 0.05 for $\mathrm{pACC} / \mathrm{ACC}$ ), with no change in the abundance of the nonphosphorylated species (Fig. 4D,E).

We then performed mSOD1 toxicity assays, wherein cultures were coinfected with either HSV-WT SOD1 or G37R mSOD1 plus either dnAMPK, WT AMPK, or Lac-Z. By ANOVA, significant groups were found $\left(F_{(5,88)}=4.55 ; p \leq 0.001\right)$. Mixed cultures expressing mSOD1 + Lac-Z showed $\sim 60 \%$ survival in SMI-32 $(+)$ neurons $(p<0.002$ compared to WT SOD1 plus Lac-Z). Remarkably, cultures coinfected with mSOD1 plus dnAMPK showed close to $100 \%$ survival $(p<0.04$ compared to mSOD1 plus Lac-Z; three independent experiments) (Fig. $4 F$ ). Coinfection of mSOD1 plus WT AMPK had no effects on survival and also showed a trend toward decreased motor neuron survival ( $p=0.09$ compared to mSOD1 plus dnAMPK). Coinfection of WT SOD1 with Lac-Z, WT AMPK, or dnAMPK had no effects on survival.

In sum, we have shown that bioenergetic defects are found in in vitro and in vivo models of $\mathrm{mSOD} 1$, and that these defects are associated with increased AMPK activity. Inhibiting AMPK activity, pharmacologically using the antagonist Compound $\mathrm{C}$ or genetically using a dominant negative construct, is neuroprotective in mSOD1-infected mixed spinal cord cultures.

\section{Placing the G85R mSOD1 C. elegans mutant into the AMPK} null strain aak-2(ok524) improves locomotor activity

To investigate the role of AMPK in an in vivo model of mSOD1 toxicity, we used a nematode $C$. elegans engineered to express human WT SOD1 and mSOD1 exclusively in neurons $\left(P_{\text {snb-1 } 1}:: W T\right.$ SOD1-YFP and $P_{\text {snb- } 1}:: G 85 R$ SOD1-YFP). We will hereafter refer to these animals as WT SOD1 and G85R worms, respectively. These animals show ALS-like pathology, such as locomotor defects and protein aggregation (Wang et al., 2009). However, these animals possess a normal lifespan, and do not show detectable neuron death (Wang et al., 2009). These animals therefore model the motor neuron dysfunction that precedes death in ALS (Gould et al., 2006; Ilieva et al., 2008). C. elegans expressing human WT SOD1 also possess mild locomotor defects compared to the N2 control strain; however, worms expressing the mutant G85R version possess far more dramatic locomotor defects (Wang et al., 2009).

Given our results that attenuating AMPK activity is beneficial in vitro, we wondered whether placing the G85R mSOD1 animal into the background of an AMPK $\alpha 2$ null animal would improve disease pathology. The gene aak-2 is the ortholog of the AMPK $\alpha 2$ subunit in nematodes, and there are several null strains available. We used the ok524 aak $\alpha 2$ deletion mutant (aak-2) and generated double mutants. We will hereafter refer to these animals as G85R;aak-2. We compared the locomotor behavior of G85R;aak-2 worms to G85R worms on two kinds of locomotor assays: a crawling assay on agar plates containing OP50 bacteria and a swimming assay. For each assay, at least two independent experiments were performed containing at least five replicates each. On the crawling assay, significant differences existed among groups during $\mathrm{L} 4\left(F_{(4,28)}=5.68 ; p<0.002\right)$ and adult stages $\left(F_{(4,22)}=7.44 ; p \leq 0.0006\right)$ (Fig. $\left.5 A, B\right)$. We observed the modest locomotor defect in WT SOD1 worms compared to the N2 con- 
trol strain at the L4 stage $(p<0.03)$, which persisted, but to a lesser degree, during the adult stage $(p=0.08)$. As anticipated, G85R worms also performed significantly worse compared to WT SOD1 counterparts during both time points. On average, G85R worms moved at only half the average speed of WT SOD1 worms during the L4 stage $(0.021 \pm 0.003 \mathrm{~mm} / \mathrm{s}$ in G85R vs $0.045 \pm 0.006 \mathrm{~mm} / \mathrm{s}$ in WT SOD 1$)$, which persisted into adulthood $(0.021 \pm$ 0.004 in $G 85 R$ vs $0.063 \pm 0.009 \mathrm{~mm} / \mathrm{s}$ in $W T$ SOD $1 ; p<0.001$ in L4 and adult worms). We also observed enhanced locomotor activity in adult aak-2 worms compared to the N2 strain; however, this effect was erased when average speed was normalized to animal size (data not shown). Double-mutant G85R;aak-2 worms demonstrated a significant improvement in average crawling speed compared to $G 85 R$ worms despite still being significantly less compared to aak-2 controls $(p<0.01$ in L4 animals and $p<$ 0.003 in adults). Enhanced locomotor behavior of double mutants was seen in L4 animals $(0.021 \pm 0.005 \mathrm{~mm} / \mathrm{s}$ in $G 85 R$ vs $0.042 \pm 0.003 \mathrm{~mm} / \mathrm{s}$ in G85R;aak-2; $p \leq$ $0.01)$ and improved to a greater degree in adult worms $(0.021 \pm 0.004 \mathrm{~mm} / \mathrm{s}$ in $G 85 R$ vs $0.09 \pm 0.007 \mathrm{~mm} / \mathrm{s}$ in G85R;aak-2; $p<$ $0.001)$. This beneficial effect also persisted until later stages of adulthood, specifically $168 \mathrm{~h}$ after synchronization, and was observed even when average speed was normalized to body size (data not shown).

We also performed a swimming assay, another test of locomotor ability, wherein we also observed group differences among $\operatorname{L4}\left(F_{(5,52)}=13.38 ; p<0.0001\right)$ and adult animals $\left(F_{(5,42)}=11.10 ; p<0.0001\right)$ (Fig. $5 C, D)$. Similar to the crawling assay, WT SOD1 worms demonstrated a mild locomotor defect compared to the N2 control worms only at the L4 stage $(p<0.002)$. There was no significant difference in aak-2 locomotor behavior compared to N2 control worms at both stages of development. However, G85R worms performed significantly worse compared to WT SOD1 counterparts during both time points such that locomotion was reduced by $>80 \%(0.042 \pm 0.005 \mathrm{~mm} / \mathrm{s}$ in G85R vs $0.271 \pm 0.032 \mathrm{~mm} / \mathrm{s}$ in $W T$ SOD1 worms during the L4 stage; $0.047 \pm 0.004 \mathrm{~mm} / \mathrm{s}$ in G85R vs $0.259 \pm 0.031 \mathrm{~mm} / \mathrm{s}$ in WT SOD1 worms during adulthood; $p<$ 0.0007 and $p<0.003$, respectively). Although G85R;aak-2 worms still performed significantly worse compared to aak-2 controls $(p<$ 0.0001 and $p<0.0001$ in L4 and adult worms, respectively), doublemutant worms revealed a significant twofold improvement in locomotor behavior compared to $G 85 R$ worms. This pattern was observed at L4 stage $(0.042 \pm 0.005 \mathrm{~mm} / \mathrm{s}$ in $G 85 R$ vs $0.120 \pm 0.013$ $\mathrm{mm} / \mathrm{s}$ in G85R;aak-2; $p<0.001)$ and persisted until adulthood $(0.047 \pm 0.004 \mathrm{~mm} / \mathrm{s}$ in $G 85 R$ vs $0.130 \pm 0.016 \mathrm{~mm} / \mathrm{s}$ in $G 85 R$; aak-2; $p<0.002)$. Furthermore, this improvement was observed as late as $168 \mathrm{~h}$ after synchronization and even when data were normalized to body size (data not shown).

We also tested the effects of genetically decreasing aak-2 activity in WT SOD1 worms to test whether its attenuation has a universally
B

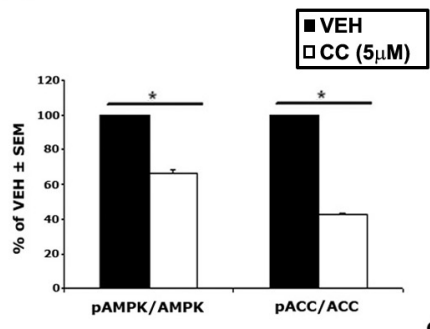

C

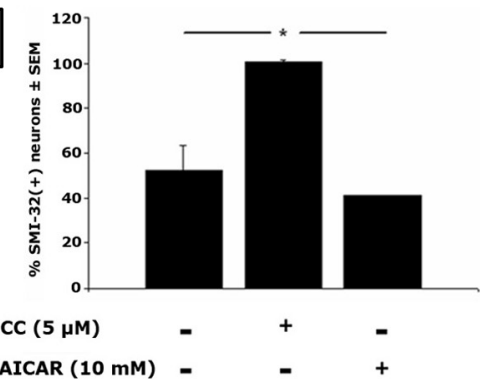

$\mathbf{E}$

$\mathbf{F}$

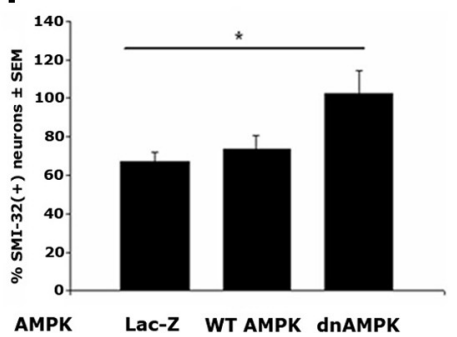

Figure 4. Inhibiting AMPK activity in vitro protects against mSOD1-induced toxicity. $\boldsymbol{A}$, Representative immunoblots of mixed

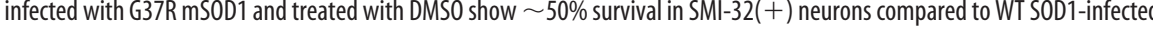

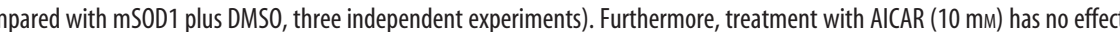
tification of immunoblots from two independent experiments. In dnAMPK-infected compared to WT AMPK-infected cultures, there is a significant decrease in the ratio of pAMPK/AMPK $\left({ }^{*} p<0.03\right)$ and a decrease in pACC/ACC $\left({ }^{*} p \leq 0.05\right)$. $\boldsymbol{F}$, In an 100\% with mSOD1 plus Lac-Z, three independent experiments). Furthermore, cultures infected with WT SOD1 plus Lac-Z, WT AMPK, or dnow no effect among groups (data not shown). $n=4$ slides per group, $4-5$ fields each. For $\boldsymbol{B}, \boldsymbol{C}, \boldsymbol{E}$, and $\boldsymbol{F}$, data are resented as percentages relative to WT SOD1-infected cultures. Asterisks indicate significance in an ANOVA, followed by post hoc tests when necessary. Error bars indicate \pm SEM.

beneficial effect on locomotor activity or if its effects are specific in the context of motor neuron disease. C. elegans expressing WT SOD1 in the aak-2(ok524) background, which we will hereafter refer to as WT SOD1;aak-2, do not show any improvement of locomotor activity on a swimming assay compared to WT SOD1 controls, and in fact even show a mild worsening effect (Fig. $5 C, D$ ). On the swimming assay, WT SOD1;aak-2 worms showed an $\sim 35 \%$ decrease in locomotor activity compared to WT SOD1 control worms. This effect shows a trend at the L4 stage $(p=0.09)$ and reaches significance in adulthood $(0.146 \pm 0.032 \mathrm{~mm} / \mathrm{s}$ in WT SOD1;aak-2 animals vs $0.259 \pm 0.031 \mathrm{~mm} / \mathrm{s}$ in WT SOD1 animals; $p<0.04)$. Similar results were observed when data were normalized to body size (data not shown). Therefore, the beneficial effect of reducing aak-2 activity appears specific to an ALS-causing mutation. Elimination of aak-2 modestly worsens locomotor behavior in young adult animals expressing a wild-type version of SOD1. This improvement was observed when data were normalized to body size (data not shown). This is consistent with previous studies reporting that metformin, a drug that increases aak-2 activity, promotes health span and locomotor behavior in normal worms (Onken and Driscoll, 2010).

To consolidate the above observations, we studied a second independently generated $P_{\text {snb } 1}:: G 85 R$ SOD1-YFP strain, termed line 18 , which will hereafter be referred to as $G 85 R(18)$. The 
A

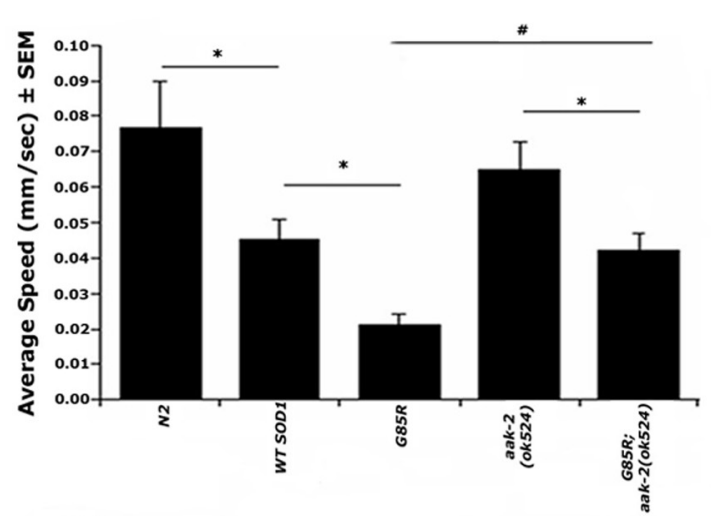

C

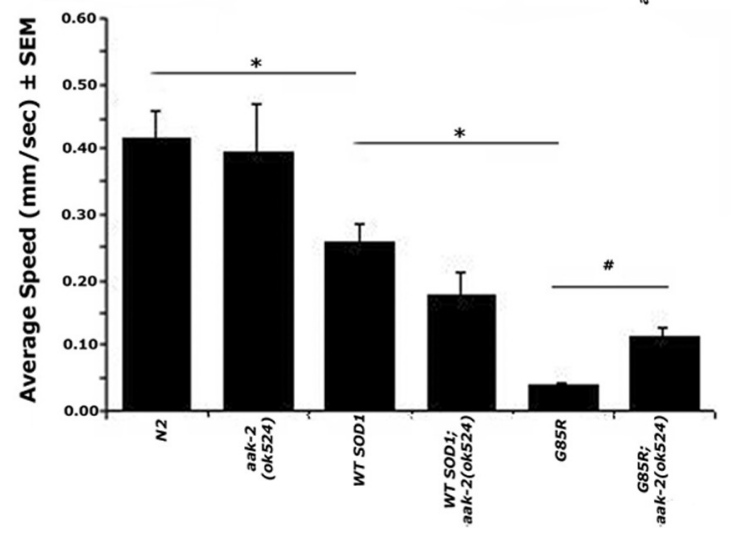

E

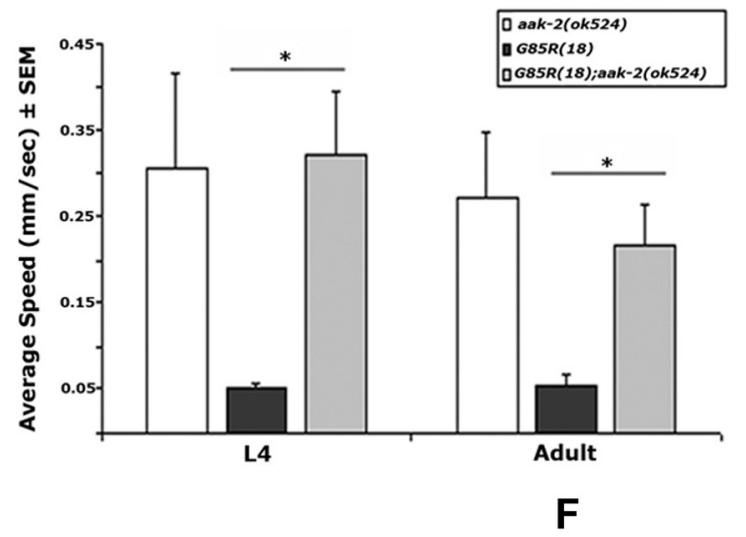

Crawling

Swimming

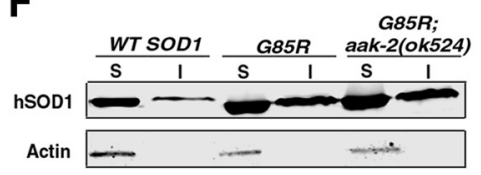

G
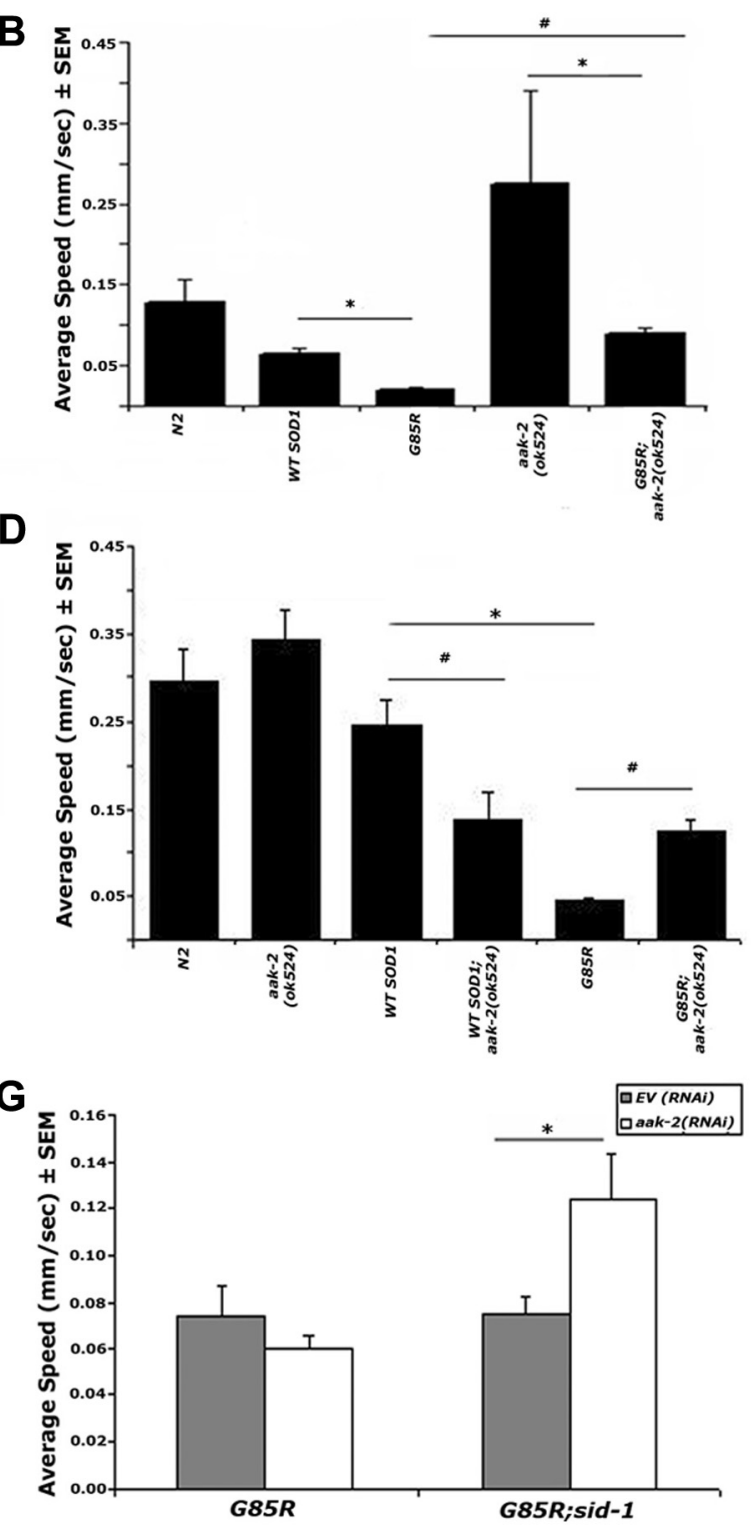

Figure 5. Placing G85RmSOD1 worms in an AMPK $\alpha 2$ (aak-2) null background improves locomotor behavior. A, In a crawling assay, L4 WT SOD1 worms exhibit decreased locomotor behavior compared to N2 worms $\left({ }^{*} p<0.03\right)$, while G85RmSOD1 worms exhibitan even more severe locomotor defect compared to WTSOD1 animals ( $\left.{ }^{*} p<0.001\right)$. L4G85R;aak-2(ok524) double-mutant worms demonstrate a significantimprovement in crawling behavior compared to G85R counterparts ( ${ }^{*} p \leq 0.01$ ), although still impaired compared to aak-2(ok524) controls ( $\left.{ }^{*} p<0.01\right)$. B. Adult WT SOD1 worms exhibit a trend toward decreased locomotor behavior compared to N2 worms $(p=0.08)$, while G85R worms still exhibit severely impaired behavior compared to WT SOD1 animals $\left({ }^{*} p<0.001\right)$. G85R; aak-2 worms also demonstrate significantly improved crawling behavior during adulthood compared to G85R counterparts ( $\left.{ }^{\#} p<0.001\right)$, although are still impaired compared to aak-2 controls ( $\left({ }^{*} p<0.003\right)$. C, In a swimming assay, L4 WT SOD1 show expected locomotor defect compared to N2 worms ( $\left.{ }^{*} p<0.002\right)$, and G85R wormsshowan even more severelocomotordefectcompared to WTSOD1 worms $\left({ }^{*} p<0.0007\right)$.L4G85R;aak-2animals displayimproved swimming behavior compared to G85R counterparts $\left({ }^{\#} p<0.001\right)$, although still performed worse compared to aak-2 controls $(p<0.0001)$. There is a trend toward decreased swimming activity in WT SOD1; aak-2(ok524) compared to WTSOD1 animals $(p=0.09)$. D, Like in $B$, WT SOD1 worms do not exhibit significant locomotor impairment compared to N2 animals; however, G85R worms show a severe locomotor phenotype compared to WT SOD1 worms ( $\left.{ }^{*} p<0.003\right)$. The significant improvementinswimming behavior of G85R;aak-2worms compared to G85R counterpartspersistsuntiladulthood ( ${ }^{*} p<0.002$ ), although G85R;aak-2 doublemutantsstill performed worsecompared to aak-2 controls ( $p<$ 0.0001). In contrast, WT SOD1; aak-2 worms exhibit significantly decreased swimming activity compared to WT SOD1 animals ( $p<0.04)$. E, Another G85R mutant line [G85R(18)], which expresses the mSOD1 transgene at a higher copy number compared to the previously used G85R mutants, also shows rescue of locomotor activity when placed in the background of the aak-2(0k524) worm [ ${ }^{*} p \leq 0.03$ and ${ }^{*} p \leq 0.04$, significantly different between G85R(18); aak-2 and G85R(18) worms in the L4and adultstages, respectively]. F, There is a decreased ratio of soluble (S) to insoluble (I) human SOD1 protein in both G85R and G85R;aak-2 worm lysates compared to WT SOD1 controls. Furthermore, there is no difference in the S/ISOD1 protein ratio between the two mutant groups. G, RNAi knockdown of aak-2 by feeding in G85R worms has no effect, but has a significant effect in the background of a sensitized RNAi mutant strain ( ${ }^{*} p \leq 0.04$ in G85R;sid- 7 worms fed aak-2 RNAi compared to EV RNAi). For $A-E$ and $G$, asterisks indicate significance between mutant strains and appropriate controls, whereas hash marks in A-D indicate significance between G85R and G85R; aak-2 animals or WTSOD1 and WT SOD1; a ak2.A-D are representative figures, and at least two other independent experiments were performed separately. For $E$ and $G$, each bar graph represents the average data from three independent experiments. For all locomotor assays, each bar graph represents data from 5-12 replicates, containing $\sim 10$ animals per group, for a total of 50-120 tested animals. Error bars indicate \pm SEM. 
G85R(18) worm expresses the mSOD1 transgene at higher copy number compared to the $G 85 R$ line used in previous experiments. To test whether or not reducing aak-2 activity will rescue an even more severe disease phenotype, we created $G 85 R(18)$; aak-2(ok524) double mutants. We then subjected aak-2(ok524), $G 85 R(18)$, and the $G 85 R(18)$;aak-2 worms to the swimming assay and observed group differences at both the $\mathrm{L} 4\left(F_{(2,17)}=3.07 ; p=\right.$ $0.07)$ and adult stages $\left(F_{(2,17)}=3.55 ; p=0.05\right)$ (Fig. 5E). G85R(18); aak-2 worms demonstrated restoration of locomotor behavior as early as L4 $(0.052 \pm 0.003 \mathrm{~mm} / \mathrm{s}$ in $G 85 R(18)$ worms vs $0.322 \pm$ $0.073 \mathrm{~mm} / \mathrm{s}$ in $G 85 R(18)$;aak-2 double mutants; $p \leq 0.03)$, and this beneficial effect persisted until adulthood $(0.055 \pm 0.01 \mathrm{~mm} / \mathrm{s}$ in G85R(18) worms vs $0.218 \pm 0.047 \mathrm{~mm} / \mathrm{s}$ in G85R(18);aak-2 worms; $p \leq 0.04)$. The percentage of improvement observed upon decreasing aak-2 activity in the $G 85 R(18)$ worms was even greater compared to that in the $G 85 R$ worms used originally [for example, compare an approximate threefold improvement in L4 G85R;aak-2 worms with an approximate sixfold improvement in L4 G85R(18);aak-2 worms]. This improvement was observed when data were normalized to body size (data not shown).

\section{There is no difference in levels of soluble and insoluble SOD1 protein in G85R and G85R;aak-2 double mutants}

In models of neurodegenerative disease, including the $G 85 R$ mSOD1 worm, aggregation of proteins such as SOD1 have been observed (Gidalevitz et al., 2009; Wang et al., 2009). We therefore asked whether or not the observed protective effect of our genetic manipulation is associated with reduced SOD1 aggregation in the double mutants. There was no difference in the total SOD1 protein content or the ratio of soluble to insoluble SOD1 protein in G85R; aak-2 worm lysates compared to $G 85 R$ worm lysates (Fig. $5 F$ ). In contrast, lysates prepared from WT SOD1 worms showed very little presence of insoluble human SOD1 compared to the soluble protein. This result suggests that the protective effects of reduced AMPK activity in the MSOD1 worm model are not associated with decreased SOD1 protein aggregation.

\section{RNAi knockdown of $a a k-2$ in neurons improves locomotor behavior}

Another method to decrease gene expression in C. elegans is through RNAi. C. elegans are unique in their ability to exhibit systemic RNAi knockdown such that RNAi bacterial clones can be fed directly to worms to induce silencing of a targeted gene (Timmons and Fire, 1998; Calixto et al., 2010). To our surprise, G85R worms fed an RNAi clone to aak-2 did not show significant improvement in swimming behavior compared to empty vector (EV) RNAi (Fig. 5G). Neurons have been reported to be refractory to the effects of RNAi, and this has been attributed to the lack of neuronal expression of SID-1, a transmembrane protein involved in the passive uptake of dsRNA (Winston et al., 2002; Feinberg and Hunter, 2003; Shih and Hunter, 2011). When sid-1 is expressed in neurons, neurons then respond to RNAi knockdown (Calixto et al., 2010). Furthermore, when sid-1 is placed into the background of a sid-1 null (pk3321) mutant containing a missense allele, the effect of neuronal RNAi is enhanced even further (Calixto et al., 2010). We therefore suspected that RNAi knockdown in the G85R did not affect neuronal expression of aak-2. In support of this, we used an AAK-2::GFP translational reporter worm (Lee et al., 2008) and subjected these animals to RNAi knockdown of aak-2. Consistent with our suspicions, AAK-2 expression was reduced in other tissues, such as the intestine and vulva, but GFP expression remained strong in neuronal tissues, particularly in the nerve ring (data not shown).
We next generated triple-mutant animals: G85R animals expressing sid-1 in neurons in the background of a sid-1 null (pk3321), hereafter termed G85R;sid-1 animals. G85R;sid-1 animals exposed to aak-2 RNAi displayed improved swimming behavior compared to worms exposed to EV RNAi $(0.124 \pm 0.014$ $\mathrm{mm} / \mathrm{s}$ vs $0.075 \pm 0.006 \mathrm{~mm} / \mathrm{s}$, respectively; $F_{(1,4)}=9.58 ; p \leq 0.04$; three independent experiments) (Fig. $5 G$ ). This effect occurred only when $G 85 R$ animals were in the sid-1 background, as shown by a significant interaction effect between genotype (G85R vs G85R;sid-1) and RNAi clone (EV vs aak-2; $F_{(1,6)}=5.99 ; p \leq$ $0.05)$. Similar results were seen when average speed was normalized to body size. In summary, these data suggest that reduced expression of aak-2 in neurons contributes to its beneficial effects in improving locomotor behavior.

\section{G85R worms exhibit fecundity defects, which are rescued by the aak-2 null background}

Reproduction and organismal intermediary metabolism are intimately linked (Cardozo et al., 2011; Donato et al., 2011). In nematodes, hermaphrodite reproduction is a complex process that involves the mobilization of lipids to generate, fertilize, and maintain oocytes and developing embryos (Kubagawa et al., 2006; Han et al., 2010). Interestingly, female G93A mSOD1 mice exhibit both bioenergetic abnormalities (Dupuis et al., 2004; Fergani et al., 2007), as well as decreased fertility (Jackson Laboratory) (Lim and Kalb, unpublished observations). In light of this, we wondered whether mSOD1 worms displayed reproductive abnormalities and, if so, whether ablation of aak-2 modified this phenotype. Accordingly, we assessed brood size, reproductive span, and sterility in our mutant worms. A dramatic $50 \%$ of $G 85 R$ worms were sterile, compared to $0 \%$ in WT SOD1 worms and in N2 controls (Fig. 6A) (data not shown for N2). From the subset of worms that were fertile, there were group differences that existed across several days of the reproductive span. There was a significant interaction effect between day of reproductive span and genotype $\left(F_{(36,297)}=22.59 ; p<0.0001\right)$ (Fig. $\left.6 B\right)$ and the total number of viable offspring summed across the entire reproductive $\operatorname{span}\left(F_{(4,38)}=23.70 ; p<0.0001\right)$ (Fig. 6C). Self-fertilized WT SOD1 hermaphrodites showed a modest decrease in the total number of viable progeny compared to N2 control worms $(p<$ $0.001)$. In contrast, $G 85 R$ worms exhibited far more severe fecundity defects. $G 85 R$ worms demonstrated a striking decrease in viable progeny, especially during Days 1 and 2 of adulthood, when normal hermaphrodites are most fertile $(p<0.002$ and $p \leq$ 0.003 compared to WT SOD1 worms for Days 1 and 2, respectively) (Fig. 6B). Moreover, G85R worms possessed less than half the number of viable progeny compared to WT SOD1 controls across the entire reproductive span (42.22 \pm 11.80 live offspring from $G 85 R$ worms vs $133.90 \pm 13.60$ live offspring from $W T$ SOD1 worms; $p<0.002$ ) (Fig. $6 C$ ). Compared to N2 controls, there were no significant differences in the average reproductive span of WT SOD1 and G85R worms ( $4.2 \pm 0.18 \mathrm{~d}$ in N2 worms vs $5.0 \pm 0.53 \mathrm{~d}$ in WT SOD1 worms vs $4.44 \pm 0.67 \mathrm{~d}$ in $G 85 R$ worms).

Several of the worms laid eggs that did not mature into viable offspring. Of the eggs laid by G85R animals, 20-50\% remained unhatched, in contrast to $0 \%$ in WT SOD1 controls during the first $5 \mathrm{~d}$ of active reproduction (Fig. 6C). Dead or unfertilized eggs occur at the end of the reproductive period due to the finite amount of sperm produced in C. elegans hermaphrodites. Unfertilized eggs were observed a day earlier in $G 85 R$ worms, such that $>90 \%$ of eggs laid by G85R worms on Day 6 were unfertilized, 

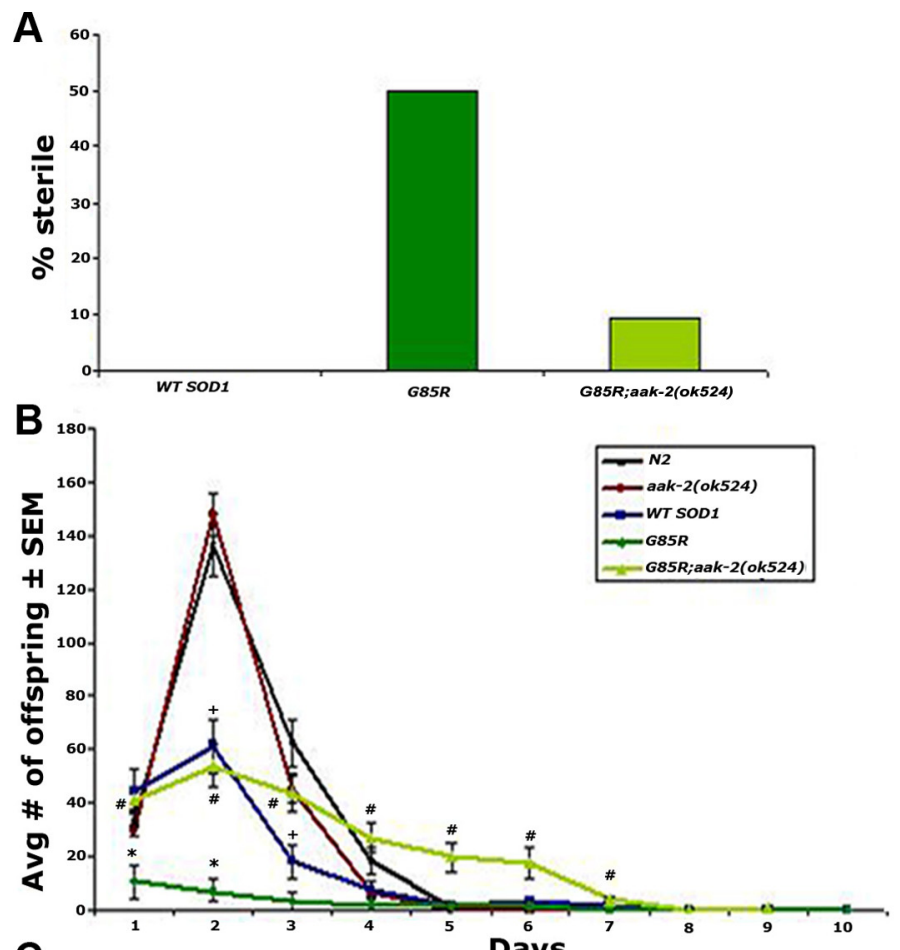

C

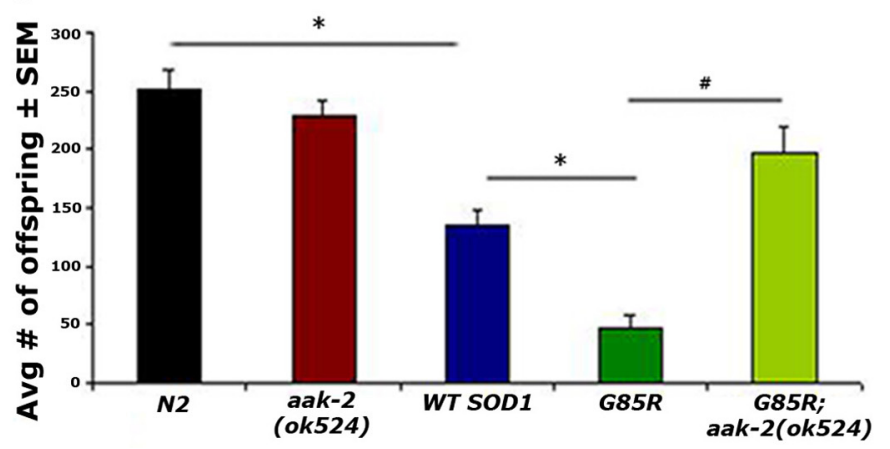

D
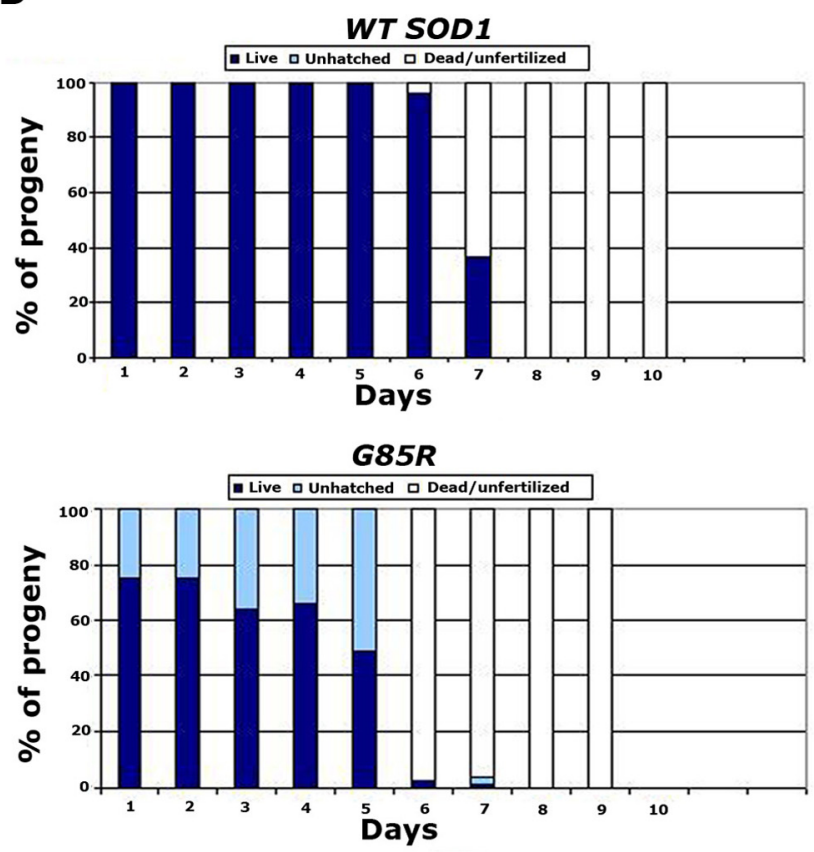

G85R;aak-2(ok524)

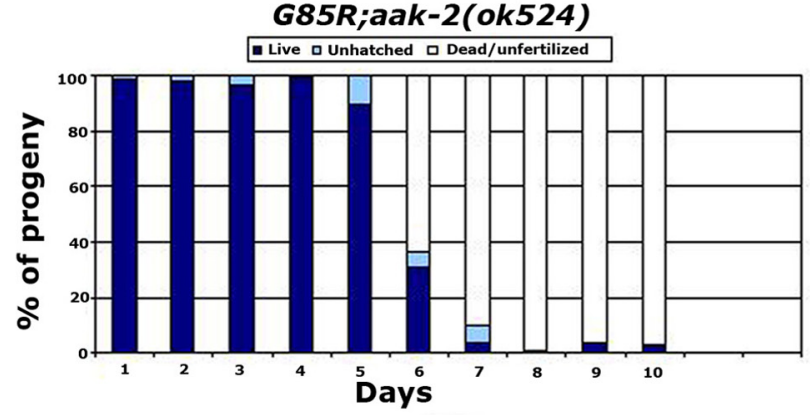

Figure 6. G85R worms exhibit fecundity defects, which are rescued by ablating aak-2. A, Approximately $50 \%$ of G85R worms are sterile compared to $0 \%$ in WT SOD1 controls and $<10 \%$ in G85R;aak-2 worms. B, Number of viable offspring for each genotype, broken down per day. Compared to N2 controls, self-fertilized WT SOD1 hermaphrodites demonstrate decreased numbers of viable progeny laid throughout the reproductive span, especially during Days 2 and $3\left({ }^{+} p<0.0001\right.$ for both days). From the subset of worms that were fertile, G85R animals demonstrate a dramatic reduction of viable offspring compared to WTSOD 1 controls, especially during Days 1 and 2 of active reproduction ( ${ }^{*} p<0.002$ and ${ }^{*} p \leq 0.003$, respectively). Placing the G85R mSOD1 animals into the aak-2(ok524) null background rescues this fecundity defect, through out the majority of the reproductive span (Days 1 through 7; $\# \leq 0.02$ ). There are no differences in brood sizes between aak-2 (ok524) animals and N2 controls. C, Sum of viable offspring through out the entire reproductive span. There is a decrease in the total number of viable offspring produced by WT SOD1 compared to $\mathrm{N} 2$ worms $\left({ }^{*} p<0.001\right)$. However, G85R mS0D1 worms show a significantly lower number of total viable offspring compared to WT SOD1 controls $\left({ }^{*} p<0.002\right)$. This defect is rescued when G85R worms are placed into the aak-2 null background ( ${ }^{\#} p<0.0001$ G85R;aak-2 vs G85R animals). There is no difference in the total number of viable offspring during the entire reproductive span between aak-2 animals and N2 controls. D. Breakdown of dropped eggs from WT SOD1, G85R, and G85R;aak-2 animals into three categories: (1) live or viable, (2) unhatched, and (3) dead or unfertilized. When counted 2-3 d fter egg drop, a significant number of G85R eggs still remained unhatched compared to 0\% in WT SOD1 worms and 10\% in G85R;aak-2 double-mutant worms. Unfertilized embryos are also present a day earlier in G85R worms (Day 6) compared to WT S0D1 animals (Day 7). Although G85R;aak-2 worms also begin producing unfertilized embryos at Day 6, only a little over $40 \%$ of total eggs laid were dead compared to close to $100 \%$ in G85R worms. For $\boldsymbol{B}$ and $\boldsymbol{C}$, plus signs indicate significance between WT SOD1 and N2 controls, asterisks indicate significance between WT SOD1 and G85R, and hash marks indicate significance between G85R and G85R;aak-2 animals. For each genotype, $n=9-20$ hermaphrodites each. Error bars indicate \pm SEM.

compared to a little over $40 \%$ of eggs laid on Day 7 by WT SOD1 animals (Fig. 6D).

Since WT SOD1 and G85R animals only express human SOD1 in neurons and not germ cells, we wondered whether feeding abnormalities, were affecting fecundity in the mSOD1 animals. We recorded pharyngeal pumping rates in adult N2, WT SOD1, and $G 85 R$ mSOD1 worms. We found no difference in pumping rates between WT SOD1 and G85R worms (154.8 \pm 6.8 pumps per minute in $W T S O D 1$ worms vs $153.6 \pm 8.3$ pumps per minute in $G 85 R$ worms), although there was a modest decrease in contraction rates in transgenic worms compared to N2 control ani- mals, which pumps an average of $187.2 \pm 8.2$ pumps per minute $\left(F_{(2,27)}=5.73 ; p<0.009\right)$.

We next investigated the consequences of placing the $G 85 R$ animals into the background of the aak-2(ok524) null animal. Compared to N2 controls, aak-2 null animals did not show significant differences in the number of viable offspring per day (Fig. $6 \mathrm{~B})$, the total number of viable offspring across the entire reproductive span (Fig. $6 C$ ), and the total reproductive span (4.2 \pm $0.22 \mathrm{~d}$ in aak-2 animals vs $4.2 \mathrm{~d} \pm 0.18$ in $\mathrm{N} 2$ controls). Placing the $G 85 R$ animals into the background of the aak-2(ok524) null worm rescued the fecundity phenotypes observed in the $G 85 R$ 
worms. G85R;aak-2 double mutants showed deceased occurrences of sterility compared to G85R animals (9\% of G85R;aak-2 animals vs $50 \%$ of $G 85 R$ animals) (Fig. $6 A$ ). Of the worms that were fertile, G85R;aak-2 hermaphrodites produced more viable offspring compared to $G 85 R$ worms throughout most of the reproductive span, especially during Days 1, 2, and 3 of adulthood ( $p \leq 0.01$ for Days 1, 2, and 3) (Fig. 6B). Furthermore, they produced a larger total number of viable offspring compared to G85R worms (198.20 \pm 21.70 live offspring from G85R;aak-2 animals vs $47.22 \pm 11.80$ live offspring from $G 85 R$ animals; $p<$ 0.0001) (Fig. 6C). Interestingly, the reproductive span of the G85R;aak-2 animals was significantly different compared to the other groups $\left(F_{(4,48)}=4.78 ; p<0.003\right)$; G85R;aak-2 animals exhibited a longer reproductive span compared to $G 85 R$ animals $(6.4 \pm 0.54 \mathrm{~d}$ vs $4.4 \pm 0.67 \mathrm{~d}$, respectively; $p<0.003)$.

Out of the total eggs laid by G85R; aak-2 worms, only $\sim 10 \%$ of these remained unhatched across the first $5 \mathrm{~d}$ of active reproduction compared to $20-50 \%$ in $G 85 R$ animals (Fig. $6 D$ ). Similar to G85R worms, G85R;aak-2 worms started laying unfertilized eggs at Day 6, but laid a smaller percentage ( $40 \%$ of total eggs laid by G85R;aak-2 were unfertilized vs close to $100 \%$ in $G 85 R$ worms). Altogether, these results indicate that $G 85 R$ animals possess severe reproductive defects that, like locomotion, are largely rescued by reducing aak-2 activity.

\section{Decreasing aak-2 activity in the MUT TDP-43(M337V) model} in C. elegans also improves locomotor behavior

Are the beneficial effects of attenuating aak-2 activity specific to the mSOD1 model or is this effect applicable to other models of motor neuron disease? To answer this question, we used another $C$. elegans model of motor neuron disease, worms engineered to express human wild-type or mutant versions of TDP-43 exclusively in neurons $\left[P_{\text {snb- } 1}:: T D P-43(W T)\right.$ and $\left.P_{\text {snb-1 }}:: T D P-43(M 337 \mathrm{~V})\right]$. We will hereafter refer to these animals as WT TDP-43 and M337V worms, respectively. Similar to G85R mSOD1 worms, $M 337 V$ mutant TDP-43 worms also display phenotypes that are reminiscent of motor neuron disease, including locomotor defects (Liachko et al., 2010). We placed the WT TDP-43 and M337V worms into the background of the aak2(ok524) null allele to generate WT TDP-43;aak-2 and M337V; aak-2 double mutants, and then tested these worms on our swimming assay. Significant differences were observed among groups during the $\operatorname{L4}\left(F_{(5,40)}=27.71 ; p \leq 0.0001\right)$ and adult stages $\left(F_{(5,43)}=33.66 ; p \leq 0.0001\right)$ (Fig. $\left.7 A, B\right)$. We observed a modest locomotor defect in WT TDP-43 worms compared to N2 worms during the L4 stage ( $p \leq 0.002$ ), but not in adult animals. We also observed the even more severe locomotor phenotype of the $M 337 \mathrm{~V}$ mutant TDP-43 worms compared to their WT TDP-43 counterparts during the L4 stage $(0.071 \pm 0.013 \mathrm{~mm} / \mathrm{s}$ vs $0.217 \pm 0.041 \mathrm{~mm} / \mathrm{s}$, respectively; $p \leq 0.002)$ and during young adulthood $(0.051 \pm 0.002 \mathrm{~mm} / \mathrm{s}$ vs $0.227 \pm 0.023 \mathrm{~mm} / \mathrm{s}$ in $M 337 V$ and $W T$ TDP-43 animals, respectively; $p \leq 0.001)$. In contrast, young adult $M 337 \mathrm{~V}$;aak-2 worms showed a $>50 \%$ improvement in locomotor behavior compared to $M 337 \mathrm{~V}$ worms $(0.082 \pm 0.011 \mathrm{~mm} / \mathrm{s}$ vs $0.051 \pm 0.002 \mathrm{~mm} / \mathrm{s}$, respectively; $p \leq$ $0.02)$, which persisted into later stages of adulthood, as late as $144 \mathrm{~h}$ after synchronization $(0.063 \pm 0.008 \mathrm{~mm} / \mathrm{s}$ in $\mathrm{M} 337 \mathrm{~V}$; aak-2 vs $0.042 \pm 0.005 \mathrm{~mm} / \mathrm{s}$ in M337V worms; $p \leq 0.05)$. This improvement was observed when data were normalized to body size (data not shown). There was no difference in locomotor behavior between WT TDP-43 and WT TDP-43;aak-2 worms both at L4 and adult stages. In summary, similar to mSOD 1 models of ALS, reducing aak-2 activity benefits another model of motor neuron disease: C. elegans overexpressing pan-neuronal human M337V mutant TDP-43. Furthermore, this beneficial effect appears specific to an ALS-causing mutation and not to transgenic worms engineered to express WT TDP-43.

\section{Decreasing aak-2 activity in the M337V mutant TDP-43 model in C. elegans does not suppress neurodegeneration in GABAergic motor neurons}

Since loss of motor neurons is a key finding in postmortem analysis of the nervous systems of ALS patients, we next wondered whether decreasing aak-2 activity is neuroprotective in vivo. Although motor neuron loss does not occur in the G85R mSOD1 animals (Wang et al., 2009), neurodegeneration in the VD- and DD-type classes of GABAergic motor neurons and a subset of dopaminergic neurons has been recorded in $M 337 \mathrm{~V}$ mutant TDP-43 animals (Liachko et al., 2010). To visualize neurons in living animals, we used the Punc-25::GFP reporter transgene driving expression of GFP in all 19 GABAergic motor neurons (Cinar et al., 2005). In a strain carrying the GFP reporter alone (CZ1200), both the dorsal and ventral nerve cords are continuous and contain the normal complement of 19 inhibitory motor neurons (13 VD and $6 \mathrm{DD}$ GABAergic neurons). We generated Punc-25::GFP; TDP-43(M337V) and Punc-25::GFP; TDP43(M337V);aak-2(ok524), hereafter referred to as M337V and M337V;aak-2 worms, respectively. M337V mutant TDP-43 worms exhibit profound degeneration of the GABAergic motor neuron network (Liachko et al., 2010). At L4 stage, both M337V and $M 337 \mathrm{~V}$;aak-2 animals displayed significant neuronal loss compared to CZ1200 control worms $\left(F_{(2,57)}=28.92 ; p<0.0001\right)$ (Fig. 7C). Furthermore, ablation of aak-2 did not significantly alter neuronal loss seen in $M 337 \mathrm{~V}$ transgenic animals at this time point $(2.1 \pm 0.25$ lost neurons in $\mathrm{M} 337 \mathrm{~V}$ vs $1.85 \pm 0.25$ lost neurons in M337V;aak-2; $<<0.40$ ) (Fig. 7C). At the young adult stage, a time point wherein improved locomotor function was observed in M337V;aak-2 animals, significant neuronal loss was still observed in both worms expressing the M337V mutation compared to CZ1200 controls $\left(F_{(2,48)}=25.29 ; p<0.0001\right)$ (Fig. $7 C)$. Moreover, no differences were observed in the number of labeled GABAergic neuronal cell bodies between M337V and M337V;aak-2 worms ( $3.75 \pm 0.46$ lost neurons vs $4.45 \pm 0.36$ lost neurons, respectively; $p=0.19$ ) (Fig. 7C). There were no detectable differences in the number of dorsal and ventral nerve cord gaps in L4 as well as young adult animals (data not shown). These observations suggest that the loss of aak-2 ameliorates motor dysfunction in the M337V mutant TDP-43 worm, but does not prevent their ultimate demise.

\section{Decreasing TBC1D1/tbc-11 activity in models of motor neuron disease does alter disease progression}

Previously, Chiang et al. (2010) created conditional, postnatal murine knock-outs (KOs) of TDP-43. Upon ablation of TDP-43, the animals become hypermetabolic and die precipitously. Transcriptional profiling revealed that this phenotype is associated with decreased TBC1D1 expression in skeletal muscle. TBC1D1 is a Rab-GTPase activating protein that has been linked to obesity and its inactivation leads to a "lean" phenotype with increased glucose transport and fatty acid oxidation in tissues, especially skeletal muscle (Chadt et al., 2008; Frosig et al., 2010). TBC1D1 is also known to be phosphorylated at serine 237 by AMPK (Chavez et al., 2008), although the functional significance of AMPKdependent phosphorylation on TBC1D1 activities, such as glucose transport, is unknown. Considering the metabolic similarities between mice with conditional postnatal ablation of 


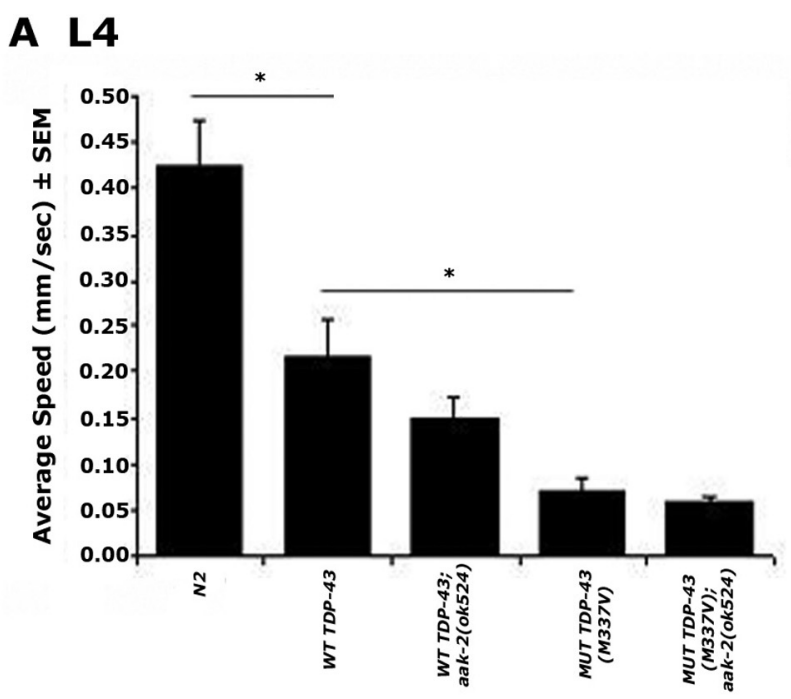

\section{B Adult}
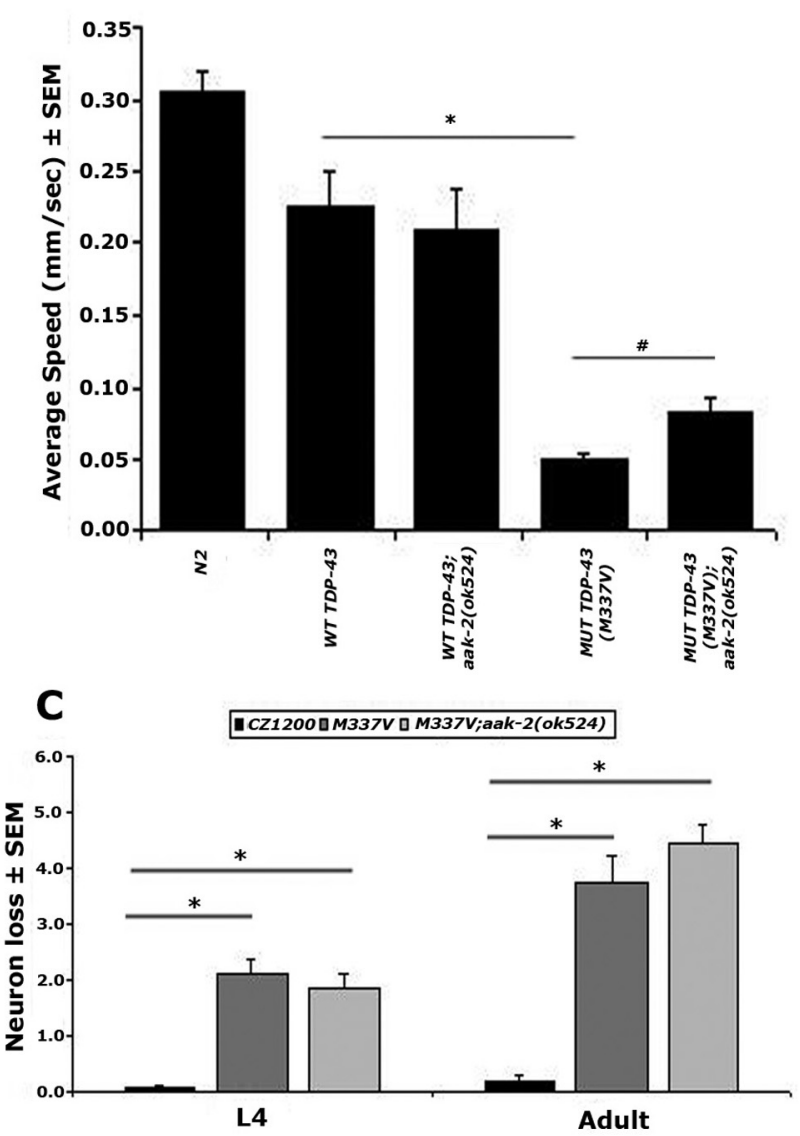

Figure 7. Decreasing aak-2 activity in the M337V mutant TDP-43 model of motor neuron disease in C. elegans also improves locomotor behavior. $\boldsymbol{A}$, In the L4 stage, WT TDP-43 worms exhibit modest locomotor defects compared to N2 worms ( ${ }^{*} p \leq 0.002$ ); however, M337V mutant TDP-43 worms exhibit a more severe locomotor defect compared to WT TDP-43 worms $\left({ }^{*} p \leq 0.002\right)$. When M337V worms were placed into the aak-2(ok524) null background, no improvement in swimming behavior was observed in the M337V;aak-2 double mutants compared to M337V controls. No difference was also detected between WT TDP-43 and WT TDP-43; aak-2 animals. $\boldsymbol{B}$, In adults, although there is no significant difference in swimming behavior between N2 and WT TDP-43 worms, there remains a severe locomotor defect between WT TDP-43 and M337V worms ( $\left.{ }^{*} p \leq 0.001\right)$. There is a significant improvement in locomotor activity in M337V;aak-2 double mutants compared to M337V worms ( ${ }^{\#} p \leq 0.02$ ), which persists until later stages of adulthood (data not shown). C, M337V mutant TDP-43 and M337V;
TDP-43 and mSOD1 transgenic mice, we wondered whether TBC1D1 activity is decreased in our mSOD1 models and whether the beneficial effects of reduced AMPK activity are associated with alterations in TBC1D1 activity.

We began by monitoring the abundance of TBC1D1 mRNA in our mSOD1 models. Mixed spinal cord cultures infected with G37R mSOD1 for $48 \mathrm{~h}$ as well as spinal cord from P120 G93A mSOD1 mice did not reveal any changes in TBC1D1 mRNA levels compared to WT SOD1-infected cultures and non-Tg control mice, respectively (Fig. $8 A$ ). Since TBC1D1 is largely expressed in skeletal muscle (Chadt et al., 2008), we also monitored transcript levels in hindlimb muscle from P120 G93A mSOD1 and non-Tg controls. No changes in transcript levels were detected in this tissue at this specific time point (Fig. $8 \mathrm{~A}$ ).

We also investigated expression levels of the worm paralog of TBC1D1, $t b c-11$, in whole-worm RNA extract from our mutant worm strains. Significant differences were detected among groups $\left(F_{(4,15)}=9.79 ; p=0.004\right)$. Compared to N2 worms, $W T$ SOD1 and $a a k-2$ worms did not exhibit any significant changes in tbc-11 mRNA levels (Fig. $8 \mathrm{~B}$ ). In contrast, there was a $70 \%$ and $50 \%$ increase in $t b c-11$ expression in young adult $G 85 R$ worms in comparison to $\mathrm{N} 2$ and $W T$ SOD1 controls, respectively ( $p=$ 0.003 compared to N2; $p<0.02$ compared to WT SOD1) (Fig. $8 \mathrm{~B})$. This increase was returned toward N2 control levels by placing the $G 85 R$ animals into the aak-2 null background ( $p=0.002$ between $G 85 R$ and $G 85 R$;aak-2 worms) (Fig. $8 B$ ). There were no differences in $t b c-11 \mathrm{mRNA}$ levels between G85R;aak-2 animals and WT SOD1 animals, as well as compared to aak-2 controls. Therefore, while TBC1D1 message is reduced in mice with conditional postnatal ablation of TDP-43, it is not reduced in the mSOD1 models we have studied.

If reduced TBC1D1 abundance contributes to the hypermetabolism seen in the conditional TDP-43 ablation mouse, one would predict that reducing $t b c-11$ in our $C$. elegans models would exacerbate disease. We pursued this issue by attenuating $t b c-11$ expression using RNAi knockdown. When G85R or G85R; sid- 1 worms were fed $t b c-11$ RNAi, no significant interaction effect was detected between genotype ( $G 85 R$ vs G85R;sid-1) and RNAi clone (EV vs $t b c-11)\left(F_{(1,10)}=0.45 ; p=0.52\right)$. Neither G85R and G85R;sid-1 animals displayed a significant worsening or improvement in locomotor behavior when $t b c-11$ was knocked down. Interestingly, there was a trend toward modest improvement in the G85R;sid-1 animals fed $t b c-11$ RNAi compared to EV RNAi $(0.122 \pm 0.017 \mathrm{~mm} / \mathrm{s}$ vs $0.086 \pm 0.009 \mathrm{~mm} / \mathrm{s}$, respectively; $F_{(1,8)}=3.41 ; p=0.10$; five independent experiments) (Fig. 8C).

Since RNAi may lead to insufficient knockdown of gene expression, we used the $t b c-11$ null strain $(o k 2576)$ and created G85R;tbc-11(ok2576) double-mutant worms, hereafter referred to as $G 85 R ; t b c-11$ worms. The $t b c-11$ null strain (ok2576) appear phenotypically wild-type and did not demonstrate any locomotor defects compared to N2 worms (data not shown). In a swimming assay, G85R; tbc-11 worms did not demonstrate any

aak-2 worms show significant loss of GABAergic neurons compared to the CZ1200 control reporter strain during $L 4$ and young adulthood $\left({ }^{*} p<0.0001\right)$. However, double-mutant $M 337 V$; aak-2 animals do not show decreased neuronal loss compared to M337V at both time points. For $A-C$, asterisks indicate significance between mutant strains and appropriate controls ( $p \leq$ 0.05 ), while hash marks indicate significance between M337V mutant TDP-43 and M337V; aak-2 controls. For $\boldsymbol{A}$ and $\boldsymbol{B}$, each bar graph represents data from 10 replicates, containing $\sim 10$ animals per group, for a total of 100 tested animals. For $C$, each graph contains data from $10-20$ worms per group. Error bars indicate \pm SEM. 

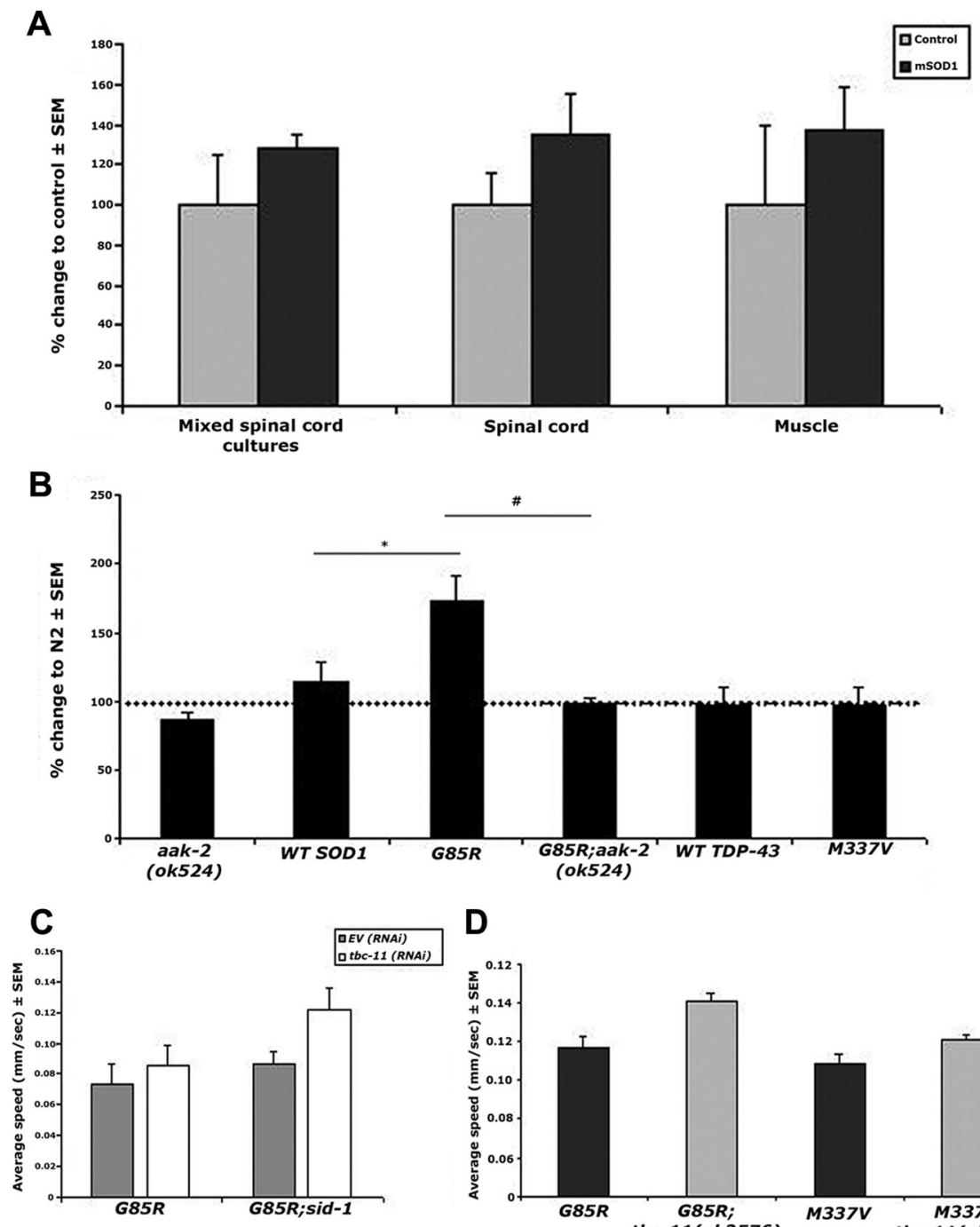

D
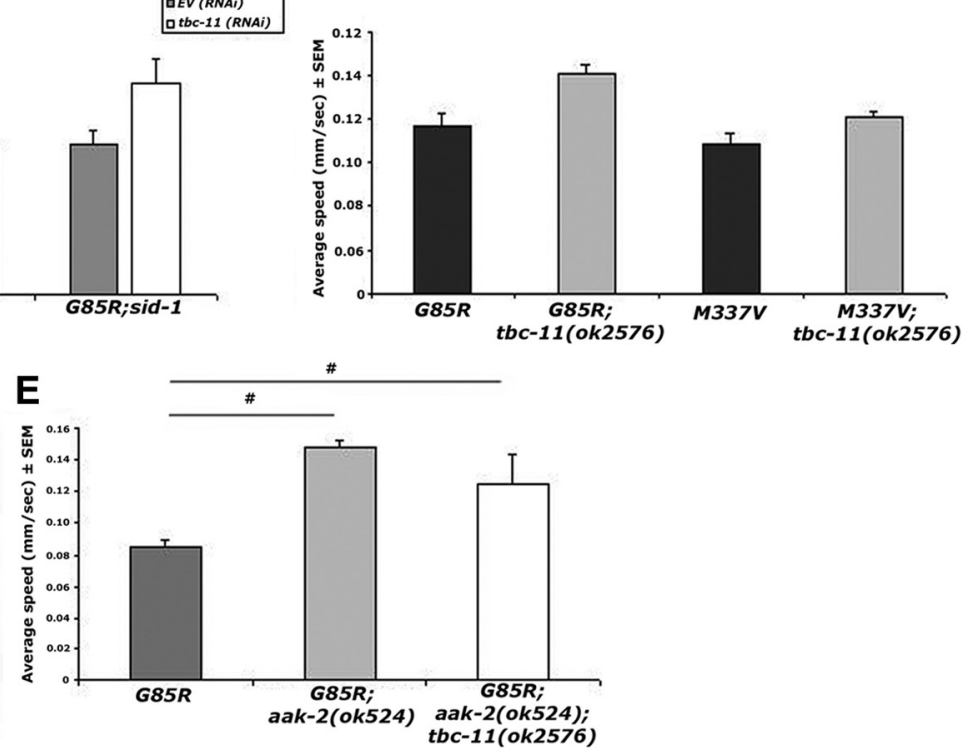

Figure 8. The relationship between TBC1D1/tbc-11 and models of motor neuron disease. $A$, Mixed spinal cord cultures infected with HSV-G37R mSOD1 for $48 \mathrm{~h}$ do not show significant changes in TBC1D1 mRNA levels compared to WT S0D1-infected cultures ( $n=3-5$ dishes per group, three independent experiments). Furthermore, spinal cord and hindlimb muscle from P120 G93A mS0D1 mice do not show altered levels of TBC1D1 compared to non-Tg controls ( $n=5-7$ animals per group). All data are normalized to $18 \mathrm{~s}$ RNA and are presented as percentages compared to controls (either HSV-WTSOD1 or non-Tg controls). B, The expression of tbc-11, the worm paralog of TBC1D1, does not differ in N2, aak-2, and WT SOD1 worms. On the contrary, young adult G85R worms show significantly elevated tbc-11 mRNA levels compared to N2 controls $(p=0.003)$ and WT SOD1 animals $\left({ }^{*} p<0.02\right)$. Placing G85R worms into the aak-2 null background normalizes tbc-11 transcript expression back to control levels ( ${ }^{~} p=0.002$ compared to G85R worms). Both young adult WT TDP-43 and M337V mutant TDP-43 worms do not show any alterations in tbc-11 mRNA expression compared to N2 controls. Representative figures are normalized to the housekeeping gene $c d c-42$ and are presented as percentages compared to $\mathrm{N} 2$ controls. At least one to two other independent experiments were performed separately. $C$, In a locomotor assay, RNAi knockdown of tbc-11 by feeding has no statistically significant effect on G85R and G85R;sid-1 worms compared to the EV control. There is a trend toward improvement in the G85R;sid-1 worms fed tbc-11 RNAi bacteria. D, G85R worms were placed into the background of a tbc-11(ok2576) null background to produce G85R;tbc-11 double-mutant worms. No significant improvement in swimming behavior is observed at the L4 time point compared to G85R controls. M337V mutant TDP-43 worms were placed into the background of a tbc-11(ok2576) null background to produce M337V;tbc-11 double-mutant worms. No significant improvement in swimming behavior is observed at the L4 time point compared to M337V controls. $\boldsymbol{E}$, There is no significant difference in swimming behavior between L4 G85R;aak-2;tbc-11 triple-mutant worms compared to G85R;aak-2 . double-mutant worms Furthermore, G85R;aak-2 and G85R;aak-2;tbc-11 worms showed a significant improvement in locomotor activity compared to G85R worms ( $p<<0.03)$. For $\boldsymbol{C}$ and $\boldsymbol{D}$, each bar graph represents the average data from three to five independent experiments. For all locomotor assays, each experiment comprises five replicates, containing $\sim 10$ animals per group, for a total of $\sim 50$ animals per experiment. Error bars indicate \pm SEM. 
significant worsening or improvement in locomotor behavior compared to $G 85 R$ counterparts $\left(F_{(1,5)}=2.02 ; p=0.23\right.$; three independent experiments) (Fig. $8 D$ ). Similar results were observed at even later stages of development (data not shown). Furthermore, similar to RNAi knockdown, there was a trend toward modest improvement in swimming behavior $(\sim 30 \%$ improvement in $G 85 R ; t b c-11$ worms compared to $G 85 R$ controls).

We next examined whether $t b c-11$ is epistatic to $a a k-2$ in our mSOD1 C. elegans by generating G85R;aak-2(ok524);tbc11(ok2576) triple-mutant worms, hereafter referred to as $G 85 R$; $a a k-2 ; t b c-11$ worms. A significant effect was observed among groups $\left(F_{(2,12)}=8.55 ; p<0.005\right)$ (Fig. $8 E$ ). Both G85R;aak-2 and G85R;aak-2;tbc-11 animals performed significantly better compared to $G 85 R$ animals $(p<0.002$ and $p<0.03$, respectively; two independent experiments). Furthermore, the locomotor behavior of G85R;aak-2;tbc-11 worms was not significantly different from G85R;aak-2 double-mutant worms. Similar results were observed at even later stages of development (data not shown). Altogether, these results indicate that in a mSOD1 model of motor neuron disease, decreased $t b c-11$ activity neither exacerbates disease progression nor genetically interacts with $a a k-2$ to alter disease progression.

We wondered whether the functional effects of altering TBC1D1/ $t b c-11$ activity may be specific to the TDP-43 model of motor neuron disease. To this end, we examined tbc-11 mRNA levels in WT TDP-43 and M337V mutant TDP-43 C. elegans models of motor neuron disease. No significant differences were detected among groups $\left(F_{(2,6)}=0.013 ; p=0.99\right)$ (Fig. $\left.8 B\right)$. There was no significant change observed in $t b c-11$ mRNA levels between WT TDP-43 and $\mathrm{N} 2$ controls $(p=0.89)$. Furthermore, WT TDP-43 and M337V worms displayed similar levels of $t b c-11$ transcript levels, which was, on average, $97 \%$ of $2 t b c-11$ transcript levels $(p<0.99)$. Similar to our results with mSOD1 animals, $t b c-11$ mRNA levels were not reduced in another model of motor neuron disease, mutations in TDP-43.

To explore the functional relevance of $t b c-11$, we placed the M337V mutant TDP-43-overexpressing worms in the background of the $t b c-11($ ok2576) null allele to generate doublemutant M337V;tbc-11(ok2576) worms, hereafter referred to as M337V;tbc-11 worms. No significant differences were detected between groups (Fig. 8D). More specifically, no significant improvement in swimming behavior was detected between $M 337 \mathrm{~V}$ and $M 337 \mathrm{~V} ; t b c-11$ worms $(0.068 \mathrm{~mm} / \mathrm{s} \pm 0.005$ in $M 337 V$ vs 0.08 $\mathrm{mm} / \mathrm{s} \pm 0.003$ in $M 337 \mathrm{~V} ; t b c-11$ worms; three independent experiments; $\left.F_{(1,5)}=5.48 ; p<0.08\right)$. This effect persisted until later stages of development (data not shown). Similar to our observations with $G 85 R$; $t b c-11$ worms, $M 337 V$;tbc-11 worms demonstrated modest improvements in locomotor behavior, compared to $M 337 \mathrm{~V}$ controls, which trended toward significance $(p<0.08)$. Altogether, these results suggest that in both mSOD1- and mutant TDP-43-overexpression models of motor neuron disease, TBC1D1/ tbc-11 does not seem to be pathophysiologically relevant.

\section{Discussion}

Both sporadic and familial ALS patients display bioenergetic abnormalities. In this population of ALS patients, indirect calorimetry reveals increased resting energy expenditure, accompanied by abnormal lipid metabolism (Kasarskis et al., 1996; Desport et al., 2005; Dupuis et al., 2008, 2011; Bouteloup et al., 2009; Funalot et al., 2009). Both G86R and G93A mice, two commonly used mSOD1 mice, as well as conditional TDP-43 KO mice, also show a hypermetabolic phenotype and compromised mitochondrial function (Dupuis et al., 2004; Chiang et al., 2010). Mitochondrial defects have been linked to increased association of the mSOD1 protein with the mitochondrial membrane and interference with the efficiency of oxidative phosphorylation (Deng et al., 2006; Ferri et al., 2006; Israelson et al., 2010; Pedrini et al., 2010). In this report, we confirm that mitochondria from cells expressing $\mathrm{mSOD} 1$ have impaired ATP production, and this is associated with activation of the energy sensor AMPK. This observation is consistent with previous literature reporting dysregulation of various metabolic genes in mSOD1 models, including fatty acid synthase, fatty acid transporter (FAT/CD36), glucose transporter 4, p53, FOX03A, mTOR, and nitric oxide synthase, all of which are downstream targets of the AMPK pathway (Gonzalez de Aguilar et al., 2000; Martin, 2000; Lukas et al., 2006; Fergani et al., 2007; Lobsiger et al., 2007; Morimoto et al., 2007; Gonzalez de Aguilar et al., 2008; Martinez et al., 2008; Mojsilovic-Petrovic et al., 2009) (Lim and Kalb, unpublished observations). Furthermore, we show that AMPK activation has adverse effects on genetic models of motor neuron disease. Our findings provide a mechanistic link between bioenergetic abnormalities and motor neuron disease.

In this study, we demonstrate that downregulating AMPK activity is beneficial in in vitro and in vivo mSOD1 models, as well as in an in vivo mutant TDP-43 model of motor neuron disease. We first establish that defects in mitochondrial ATP synthesis are associated with increased AMPK activity. When AMPK activity is decreased pharmacologically or genetically, in vitro, we observe increased motor neuron protection. In vivo, when C. elegans that possess pan-neuronal expression of G85R mSOD1 or M337V mutant TDP-43 are placed on the background of an AMPK (aak-2) null strain, we observe improved locomotor behavior. Furthermore, ablating aak-2 in G85R mSOD1 worms rescues fecundity defects. This observation is noteworthy for several reasons. Fecundity defects have been reported in ALS patients (Johnson et al., 1995), as well as mouse models of the disease. Reproductive health is also tightly regulated by the metabolic state of organisms (Cardozo et al., 2011; Donato et al., 2011). For example, female mice that are null for the adipocyte hormone leptin (ob/ob mice) are not only obese, hypometabolic, and hyperphagic, but also sterile (Chehab et al., 1996). Furthermore, reproductive span has been linked to several pathways involved in aging and metabolism (such as insulin signaling), as well as mitochondrial function (Hsin and Kenyon, 1999; Branicky et al., 2000; Luo et al., 2010). Reduced fecundity in mSOD1 models may therefore be indicative of whole organism bioenergetic abnormalities. It will be interesting to determine whether the fecundity effect of ablating aak-2 in the MSOD1 worm is a noncell-autonomous phenomenon.

Although decreasing aak-2 activity improved the locomotor defects of M337V mutant TDP-43 worms, it did not rescue neurodegeneration observed in this model. These results suggest that the beneficial effects of decreasing aak-2 activity may be through improving the functioning of neurons under conditions of stress and proteotoxicity. Several reports highlight the presence of neuronal dysfunction before neuronal death in several models of neurodegenerative disease, such as ALS (Gould et al., 2006; Ilieva et al., 2008), spinal muscular atrophy (Mentis et al., 2011), spinocerebellar ataxia (Barnes et al., 2011; Shakkottai et al., 2011), and Huntington's disease (Spampanato et al., 2008). Therefore, manipulations that may benefit neuronal function may at least be partially independent of processes that lead eventually to neuronal death in these disease models. 
What downstream targets of AMPK involved in metabolism may be dysregulated in mSOD1 models? Here, we report that expression of PGC- $1 \alpha$ message and its downstream targets are not altered in mSOD1-infected cultures, spinal cord, and hindlimb muscle from symptomatic mSOD1 mice. These results were unexpected since, typically, impaired ATP synthesis should trigger an increase in PGC- $1 \alpha$ message, protein, and transcription activity. However, it is also plausible that changes in PGC- $1 \alpha$ expression may differ between specific neuronal populations (i.e., sensory vs motor neurons) or cell-types (i.e., neurons vs astrocytes) (Cui et al., 2006) and may therefore be masked in mixed culture or whole tissue preparations. Our results do not rule out changes in PGC- $1 \alpha$ protein abundance or alterations in posttranslational modifications. More experiments are needed to investigate the relationship between bioenergetic abnormalities and its effects on AMPK and PGC- $1 \alpha$.

Another gene of interest is TBC1D1. Chiang et al. (2010) generated mice with a conditional postnatal ablation of TDP-43 (TDP-43 KO) and found that these animals develop rapid weight loss and increased energy expenditure. These abnormalities bear striking resemblance to the hypermetabolic defects observed in mSOD1 mice, although the time frame of sign and symptom progression in the TDP-43 KO mice is greatly accelerated. There is a reduction in TBC1D1 expression in the muscle of the TDP- 43 $\mathrm{KO}$ mice, and this is of interest because TBC1D1 inactivation leads to increased glucose clearance and fatty acid oxidation in muscles (Chadt et al., 2008). If TBC1D1 expression was suppressed in our mSOD1 and mutant TDP-43 models, it might account for the hypermetabolic phenotype and be pathophysiologically relevant. In mSOD1-infected mixed spinal cord cultures, as well as tissues from symptomatic mSOD1 mice, we found that TBC1D1 message was similar in comparison to controls. In fact, instead of showing decreased expression, TBC1D1 mRNA levels were even slightly elevated. A similar pattern was observed for the expression of $t b c-11$, the worm paralog of TBC1D1, in whole-worm lysates, such that $G 85 R$ worms showed significantly increased $t b c-11$ expression compared to WT SOD1 worms. No significant alteration in $t b c-11$ message levels was detected between WT TDP-43 and M337V worms. Finally, in several functional assays, we demonstrate that decreasing $t b c-11$ activity in G85R and M337V mutant worms does not exacerbate disease, and in some cases even leads to a modest improvement. In addition, epistatic experiments suggest that the beneficial effects of $a a k-2$ are independent of $t b c-11$ activity. Altogether, these results indicate that $\mathrm{TBC} 1 \mathrm{D} 1 / t b c-11$ is unlikely to participate in the bioenergetic abnormalities observed in mSOD1 mice and does not contribute to motor neuron dysfunction/death in mSOD1 or mutant TDP-43 worm models.

Why might increased AMPK activity be detrimental in models of motor neuron disease? The metabolic phenotype observed in mSOD1 and TDP-43 models matches the outcomes one may anticipate when AMPK is activated, including increased fatty acid oxidation and glucose clearance. The physiological characterization of these models appears to be that of a "starvation" phenotype, wherein ATP levels are low and fat stores are rapidly depleted despite normal to increased food intake. In fact, caloric restriction, which activates AMPK (Baur et al., 2006; Curtis et al., 2006), further shortens lifespan and accelerates disease progression (Hamadeh et al., 2005; Mattson et al., 2007; Patel et al., 2010). On the contrary, feeding mSOD1 mice a high-energy diet remarkably increases lifespan, improves locomotor behavior, increases fat stores, and protects motor neurons (Dupuis et al., 2004; Browne et al., 2006; Zhao et al., 2006). AMPK activation may be initially beneficial to turn on catabolic pathways that can increase ATP levels and therefore restore energy homeostasis. However, chronic AMPK activation might become detrimental when energy stores are exhausted and important anabolic pathways remain shut down. These pathways may include glucose, cholesterol, and fat synthesis pathways, as well as pathways promoting protein translation, which are all crucial for survival. Chronic AMPK activation could be particularly detrimental to neurons with high metabolic demands, such as motor neurons with their relatively large size and long axonal projections. In response to AMPK activation, motor neurons may reallocate resources away from vital anabolic processes, such as axonal transport, as well as protein and lipid synthesis. Over the long run, this may lead to dysfunction and eventually neuronal death.

Previous reports support that excessive AMPK activity may have detrimental effects in stressed conditions. In mouse models of ischemia, AMPK activation has been observed (McCullough et al., 2005; Li et al., 2007; Mukherjee et al., 2008). More importantly, pharmacological and genetic reduction of AMPK has been shown to be neuroprotective in the ischemic mouse brain. In Alzheimer's disease, AMPK activation leads to increased production of $\mathrm{A} \beta$ peptides, which contribute to amyloid plaque formation (Chen et al., 2009). In response to glucose deprivation, AMPK activation may also induce apoptotic cell death through its interactions with tumor-suppressor p53 (Jones et al., 2005; Okoshi et al., 2008). It has also been shown previously that inhibition of SNF1, the AMPK homolog in yeast, promotes stress resistance and increases replicative lifespan, independent of caloric restriction pathways (Lu et al., 2011). Although AMPK activation may be beneficial in some settings, such as in the context of cardiovascular diseases, diabetes, and obesity, our data and the evidence cited above suggest that it is reasonable to think that increased and prolonged AMPK activity may paradoxically play a detrimental role in the setting of some neurodegenerative diseases.

In sum, we report the potential role that the energy sensor AMPK may play in models of motor neuron disease. AMPK may therefore provide a link connecting metabolic perturbations, motor neuron degeneration, and muscle pathology observed in ALS patients and animal models. Further experiments to support this link are underway. Decreasing AMPK activity and perhaps other pathways that lead to increased energy expenditure in genetic models of motor neuron disease may potentially be an intriguing therapeutic target to treat ALS patients.

\section{References}

Barnes JA, Ebner BA, Duvick LA, Gao W, Chen G, Orr HT, Ebner TJ (2011) Abnormalities in the climbing fiber-Purkinje cell circuitry contribute to neuronal dysfunction in ATXN1 [82Q] mice. J Neurosci 31:12778-12789.

Baur JA, Pearson KJ, Price NL, Jamieson HA, Lerin C, Kalra A, Prabhu VV, Allard JS, Lopez-Lluch G, Lewis K, Pistell PJ, Poosala S, Becker KG, Boss O, Gwinn D, Wang M, Ramaswamy S, Fishbein KW, Spencer RG, Lakatta EG (2006) Resveratrol improves health and survival of mice on a highcalorie diet. Nature 444:337-342.

Bendotti C, Calvaresi N, Chiveri L, Prelle A, Moggio M, Braga M, Silani V, De Biasi S (2001) Early vacuolization and mitochondrial damage in motor neurons of FALS mice are not associated with apoptosis or with changes in cytochrome oxidase histochemical reactivity. J Neurol Sci 191:25-33.

Bouteloup C, Desport JC, Clavelou P, Guy N, Derumeaux-Burel H, Ferrier A, Couratier P (2009) Hypermetabolism in ALS patients: an early and persistent phenomenon. J Neurol 256:1236-1242.

Branicky R, Benard C, Hekimi S (2000) Clk-1, mitochondria, and physiological rates. Bioessays 22:48-56.

Braun RJ, Sommer C, Carmona-Gutierrez D, Khoury CM, Ring J, Buttner S, Madeo F (2011) Neurotoxic 43-kDa TAR DNA-binding protein (TDP- 
43) triggers mitochondrion-dependent programmed cell death in yeast. J Biol Chem 286:19958-19972.

Browne SE, Yang L, DiMauro JP, Fuller SW, Licata SC, Beal MF (2006) Bioenergetic abnormalities in discrete cerebral motor pathways presage spinal cord pathology in the G93A SOD1 mouse model of ALS. Neurobiol Dis 22:599-610.

Calixto A, Chelur D, Topalidou I, Chen X, Chalfie M (2010) Enhanced neuronal RNAi in C. elegans using SID-1. Nat Methods 7:554-559.

Cardozo E, Pavone ME, Hirshfeld-Cytron JE (2011) Metabolic syndrome and oocyte quality. Trends Endocrinol Metab 22:103-109.

Cassina P, Cassina A, Pehar M, Castellanos R, Gandelman M, de Leon A, Robinson KM, Mason RP, Beckman JS, Barbeito L, Radi R (2008) Mitochondrial dysfunction in SOD1G93A-bearing astrocytes promotes motor neuron degeneration: Prevention by mitochondrial-targeted antioxidants. J Neurosci 28:4115-4122.

Chadt A, Leicht K, Deshmukh A, Jiang LQ, Scherneck S, Bernhardt U, Dreja T, Vogel H, Schmolz K, Kluge R, Zierath JR, Hultschig C, Hoeben RC, Schurmann A, Joost HG, Al-Hasani H (2008) Tbcldl mutation in lean mouse strain confers leanness and protects from diet-induced obesity. Nat Genet 40:1354-1359.

Chaturvedi RK, Adhihetty P, Shukla S, Hennessy T, Calingasan N, Yang L, Starkov A, Kiaei M, Cannella M, Sassone J, Ciammola A, Squitieri F, Beal MF (2009) Impaired PGC-1alpha function in muscle in Huntington's disease. Hum Mol Genet 18:3048-3065.

Chavez JA, Roach WG, Keller SR, Lane WS, Lienhard GE (2008) Inhibition of GLUT4 translocation by Tbcld1, a rab GTPase-activating protein abundant in skeletal muscle, is partially relieved by AMP-activated protein kinase activation. J Biol Chem 283:9187-9195.

Chehab FF, Lim ME, Lu R (1996) Correction of the sterility defect in homozygous obese female mice by treatment with the human recombinant leptin. Nat Genet 12:318-320.

Chen Y, Zhou K, Wang R, Liu Y, Kwak YD, Ma T, Thompson RC, Zhao Y, Smith L, Gasparini L, Luo Z, Xu H, Liao FF (2009) Antidiabetic drug metformin (GlucophageR) increases biogenesis of Alzheimer's amyloid peptides via up-regulating BACE1 transcription. Proc Natl Acad Sci U S A 106:3907-3912.

Chiang PM, Ling J, Jeong YH, Price DL, Aja SM, Wong PC (2010) Deletion of TDP-43 down-regulates Tbcld1, a gene linked to obesity, and alters body fat metabolism. Proc Natl Acad Sci U S A 107:16320-16324.

Cinar H, Keles S, Jin Y (2005) Expression profiling of GABAergic motor neurons in Caenorhabditis elegans. Curr Biol 15:340-346.

Cui L, Jeong H, Borovecki F, Parkhurst CN, Tanese N, Krainc D (2006) Transcriptional repression of PGC-1alpha by mutant huntingtin leads to mitochondrial dysfunction and neurodegeneration. Cell 127:59-69.

Curtis R, O'Connor G, DiStefano PS (2006) Aging networks in Caenorhabditis elegans: AMP-activated protein kinase (aak-2) links multiple aging and metabolism pathways. Aging Cell 5:119-126.

Deng HX, Shi Y, Furukawa Y, Zhai H, Fu R, Liu E, Gorrie GH, Khan MS, Hung WY, Bigio EH, Lukas T, Dal Canto MC, O'Halloran TV, Siddique T (2006) Conversion to the amyotrophic lateral sclerosis phenotype is associated with intermolecular linked insoluble aggregates of SOD1 in mitochondria. Proc Natl Acad Sci U S A 103:7142-7147.

Desport JC, Preux PM, Magy L, Boirie Y, Vallat JM, Beaufrere B, Couratier P (2001) Factors correlated with hypermetabolism in patients with amyotrophic lateral sclerosis. Am J Clin Nutr 74:328-334.

Desport JC, Torny F, Lacoste M, Preux PM, Couratier P (2005) Hypermetabolism in ALS: Correlations with clinical and paraclinical parameters. Neurodegener Dis 2:202-207.

Donato J Jr, Cravo RM, Frazao R, Elias CF (2011) Hypothalamic sites of leptin action linking metabolism and reproduction. Neuroendocrinology 93:9-18.

Dupuis L, Oudart H, Rene F, Gonzalez de Aguilar JL, Loeffler JP (2004) Evidence for defective energy homeostasis in amyotrophic lateral sclerosis: Benefit of a high-energy diet in a transgenic mouse model. Proc Natl Acad Sci U S A 101:11159-11164.

Dupuis L, Corcia P, Fergani A, Gonzalez De Aguilar JL, Bonnefont-Rousselot D, Bittar R, Seilhean D, Hauw JJ, Lacomblez L, Loeffler JP, Meininger V (2008) Dyslipidemia is a protective factor in amyotrophic lateral sclerosis. Neurology 70:1004-1009.

Dupuis L, Pradat PF, Ludolph AC, Loeffler JP (2011) Energy metabolism in amyotrophic lateral sclerosis. Lancet Neurol 10:75-82.
Feinberg EH, Hunter CP (2003) Transport of dsRNA into cells by the transmembrane protein SID-1. Science 301:1545-1547.

Fergani A, Oudart H, Gonzalez De Aguilar JL, Fricker B, Rene F, Hocquette JF, Meininger V, Dupuis L, Loeffler JP (2007) Increased peripheral lipid clearance in an animal model of amyotrophic lateral sclerosis. J Lipid Res 48:1571-1580.

Ferri A, Cozzolino M, Crosio C, Nencini M, Casciati A, Gralla EB, Rotilio G, Valentine JS, Carri MT (2006) Familial ALS-superoxide dismutases associate with mitochondria and shift their redox potentials. Proc Natl Acad Sci U S A 103:13860-13865.

Finck BN, Kelly DP (2006) PGC-1 coactivators: Inducible regulators of energy metabolism in health and disease. J Clin Invest 116:615-622.

Fraser AG, Kamath RS, Zipperlen P, Martinez-Campos M, Sohrmann M, Ahringer J (2000) Functional genomic analysis of C. elegans chromosome I by systematic RNA interference. Nature 408:325-330.

Frosig C, Pehmoller C, Birk JB, Richter EA, Wojtaszewski JF (2010) Exercise-induced TBC1D1 Ser237 phosphorylation and 14-3-3 protein binding capacity in human skeletal muscle. J Physiol 588:4539-4548.

Funalot B, Desport JC, Sturtz F, Camu W, Couratier P (2009) High metabolic level in patients with familial amyotrophic lateral sclerosis. Amyotroph Lateral Scler 10:113-117.

Gidalevitz T, Krupinski T, Garcia S, Morimoto RI (2009) Destabilizing protein polymorphisms in the genetic background direct phenotypic expression of mutant SOD1 toxicity. PLoS Genet 5:e1000399.

Gonzalez de Aguilar JL, Gordon JW, Rene F, de Tapia M, Lutz-Bucher B, Gaiddon C, Loeffler JP (2000) Alteration of the bcl-x/Bax ratio in a transgenic mouse model of amyotrophic lateral sclerosis: evidence for the implication of the p53 signaling pathway. Neurobiol Dis 7:406-415.

Gonzalez de Aguilar JL, Niederhauser-Wiederkehr C, Halter B, De Tapia M, Di Scala F, Demougin P, Dupuis L, Primig M, Meininger V, Loeffler JP (2008) Gene profiling of skeletal muscle in an amyotrophic lateral sclerosis mouse model. Physiol Genomics 32:207-218.

Gould TW, Buss RR, Vinsant S, Prevette D, Sun W, Knudson CM, Milligan CE, Oppenheim RW (2006) Complete dissociation of motor neuron death from motor dysfunction by bax deletion in a mouse model of ALS. J Neurosci 26:8774-8786.

Hamadeh MJ, Rodriguez MC, Kaczor JJ, Tarnopolsky MA (2005) Caloric restriction transiently improves motor performance but hastens clinical onset of disease in the $\mathrm{Cu} / \mathrm{Zn}$-superoxide dismutase mutant $\mathrm{G} 93 \mathrm{~A}$ mouse. Muscle Nerve 31:214-220.

Han SM, Cottee PA, Miller MA (2010) Sperm and oocyte communication mechanisms controlling C. elegans fertility. Dev Dyn 239:1265-1281.

Hardie DG (2007) AMP-activated/SNF1 protein kinases: conserved guardians of cellular energy. Nat Rev Mol Cell Biol 8:774-785.

Hathorn T, Snyder-Keller A, Messer A (2011) Nicotinamide improves motor deficits and upregulates PGC-1alpha and BDNF gene expression in a mouse model of Huntington's disease. Neurobiol Dis 41:43-50.

Hsin H, Kenyon C (1999) Signals from the reproductive system regulate the lifespan of C. elegans. Nature 399:362-366.

Ilieva HS, Yamanaka K, Malkmus S, Kakinohana O, Yaksh T, Marsala M, Cleveland DW (2008) Mutant dynein (loa) triggers proprioceptive axon loss that extends survival only in the SOD1 ALS model with highest motor neuron death. Proc Natl Acad Sci U S A 105:12599-12604.

Irrcher I, Ljubicic V, Kirwan AF, Hood DA (2008) AMP-activated protein kinase-regulated activation of the PGC-1alpha promoter in skeletal muscle cells. PLoS One 3:e3614.

Israelson A, Arbel N, Da Cruz S, Ilieva H, Yamanaka K, Shoshan-Barmatz V, Cleveland DW (2010) Misfolded mutant SOD1 directly inhibits VDAC1 conductance in a mouse model of inherited ALS. Neuron 67:575-587.

Jager S, Handschin C, St-Pierre J, Spiegelman BM (2007) AMP-activated protein kinase (AMPK) action in skeletal muscle via direct phosphorylation of PGC-1alpha. Proc Natl Acad Sci U S A 104:12017-12022.

Jekabsons MB, Nicholls DG (2004) In situ respiration and bioenergetic status of mitochondria in primary cerebellar granule neuronal cultures exposed continuously to glutamate. J Biol Chem 279:32989-33000.

Jeong GB, Werner M, Gazula VR, Itoh T, Roberts M, David S, Pfister B, Cohen A, Neve RL, Hollmann M, Kalb R (2006) Bi-directional control of motor neuron dendrite remodeling by the calcium permeability of AMPA receptors. Mol Cell Neurosci 32:299-314.

Johnson WG, Lucek PR, Chatkupt S, Furman Y, Lustenberger A, Lazzarini A 
(1995) Reduced fecundity in male ALS gene-carriers. Am J Med Genet 59:149-153.

Jones RG, Plas DR, Kubek S, Buzzai M, Mu J, Xu Y, Birnbaum MJ, Thompson CB (2005) AMP-activated protein kinase induces a p53-dependent metabolic checkpoint. Mol Cell 18:283-293.

Kahn BB, Alquier T, Carling D, Hardie DG (2005) AMP-activated protein kinase: Ancient energy gauge provides clues to modern understanding of metabolism. Cell Metab 1:15-25.

Kasarskis EJ, Berryman S, Vanderleest JG, Schneider AR, McClain CJ (1996) Nutritional status of patients with amyotrophic lateral sclerosis: Relation to the proximity of death. Am J Clin Nutr 63:130-137.

Kim SM, Kim H, Kim JE, Park KS, Sung JJ, Kim SH, Lee KW (2011) Amyotrophic lateral sclerosis is associated with hypolipidemia at the presymptomatic stage in mice. PLoS One 6:e17985.

Kubagawa HM, Watts JL, Corrigan C, Edmonds JW, Sztul E, Browse J, Miller MA (2006) Oocyte signals derived from polyunsaturated fatty acids control sperm recruitment in vivo. Nat Cell Biol 8:1143-1148.

Lee H, Cho JS, Lambacher N, Lee J, Lee SJ, Lee TH, Gartner A, Koo HS (2008) The Caenorhabditis elegans AMP-activated protein kinase AAK-2 is phosphorylated by LKB1 and is required for resistance to oxidative stress and for normal motility and foraging behavior. J Biol Chem 283:14988-14993.

Li J, Zeng Z, Viollet B, Ronnett GV, McCullough LD (2007) Neuroprotective effects of adenosine monophosphate-activated protein kinase inhibition and gene deletion in stroke. Stroke 38:2992-2999.

Liachko NF, Guthrie CR, Kraemer BC (2010) Phosphorylation promotes neurotoxicity in a Caenorhabditis elegans model of TDP-43 proteinopathy. J Neurosci 30:16208-16219.

Lobsiger CS, Boillee S, Cleveland DW (2007) Toxicity from different SOD1 mutants dysregulates the complement system and the neuronal regenerative response in ALS motor neurons. Proc Natl Acad Sci U S A 104:7319-7326.

Long YC, Zierath JR (2006) AMP-activated protein kinase signaling in metabolic regulation. J Clin Invest 116:1776-1783.

Lu JY, Lin YY, Sheu JC, Wu JT, Lee FJ, Chen Y, Lin MI, Chiang FT, Tai TY, Berger SL, Zhao Y, Tsai KS, Zhu H, Chuang LM, Boeke JD (2011) Acetylation of yeast AMPK controls intrinsic aging independently of caloric restriction. Cell 146:969-979.

Lukas TJ, Luo WW, Mao H, Cole N, Siddique T (2006) Informatics-assisted protein profiling in a transgenic mouse model of amyotrophic lateral sclerosis. Mol Cell Proteomics 5:1233-1244.

Luo S, Kleemann GA, Ashraf JM, Shaw WM, Murphy CT (2010) TGF-beta and insulin signaling regulate reproductive aging via oocyte and germline quality maintenance. Cell 143:299-312.

Martin LJ (2000) p53 is abnormally elevated and active in the CNS of patients with amyotrophic lateral sclerosis. Neurobiol Dis 7:613-622.

Martinez JA, Francis GJ, Liu WQ, Pradzinsky N, Fine J, Wilson M, Hanson LR, Frey WH II, Zochodne D, Gordon T, Toth C (2008) Intranasal delivery of insulin and a nitric oxide synthase inhibitor in an experimental model of amyotrophic lateral sclerosis. Neuroscience 157:908-925.

Mattiazzi M, D’Aurelio M, Gajewski CD, Martushova K, Kiaei M, Beal MF, Manfredi G (2002) Mutated human SOD1 causes dysfunction of oxidative phosphorylation in mitochondria of transgenic mice. J Biol Chem 277:29626-29633.

Mattson MP, Cutler RG, Camandola S (2007) Energy intake and amyotrophic lateral sclerosis. Neuromolecular Med 9:17-20.

McConoughey SJ, Basso M, Niatsetskaya ZV, Sleiman SF, Smirnova NA, Langley BC, Mahishi L, Cooper AJ, Antonyak MA, Cerione RA, Li B, Starkov A, Chaturvedi RK, Beal MF, Coppola G, Geschwind DH, Ryu H, Xia L, Iismaa SE, Pallos J, et al. (2010) Inhibition of transglutaminase 2 mitigates transcriptional dysregulation in models of Huntington disease. EMBO Mol Med 2:349-370.

McCullough LD, Zeng Z, Li H, Landree LE, McFadden J, Ronnett GV (2005) Pharmacological inhibition of AMP-activated protein kinase provides neuroprotection in stroke. J Biol Chem 280:20493-20502.

Mentis GZ, Blivis D, Liu W, Drobac E, Crowder ME, Kong L, Alvarez FJ, Sumner CJ, O'Donovan MJ (2011) Early functional impairment of sensory-motor connectivity in a mouse model of spinal muscular atrophy. Neuron 69:453-467.

Mojsilovic-Petrovic J, Jeong GB, Crocker A, Arneja A, David S, Russell DS, Kalb RG (2006) Protecting motor neurons from toxic insult by antagonism of adenosine A2a and trk receptors. J Neurosci 26:9250-9263.

Mojsilovic-Petrovic J, Nedelsky N, Boccitto M, Mano I, Georgiades SN, Zhou
W, Liu Y, Neve RL, Taylor JP, Driscoll M, Clardy J, Merry D, Kalb RG (2009) FOXO3a is broadly neuroprotective in vitro and in vivo against insults implicated in motor neuron diseases. J Neurosci 29:8236-8247.

Morimoto N, Nagai M, Ohta Y, Miyazaki K, Kurata T, Morimoto M, Murakami T, Takehisa Y, Ikeda Y, Kamiya T, Abe K (2007) Increased autophagy in transgenic mice with a G93A mutant SOD1 gene. Brain Res 1167:112-117.

Mu J, Brozinick JT Jr, Valladares O, Bucan M, Birnbaum MJ (2001) A role for AMP-activated protein kinase in contraction- and hypoxia-regulated glucose transport in skeletal muscle. Mol Cell 7:1085-1094.

Mukherjee P, Mulrooney TJ, Marsh J, Blair D, Chiles TC, Seyfried TN (2008) Differential effects of energy stress on AMPK phosphorylation and apoptosis in experimental brain tumor and normal brain. Mol Cancer $7: 37-52$.

Neve RL, Howe JR, Hong S, Kalb RG (1997) Introduction of the glutamate receptor subunit 1 into motor neurons in vitro and in vivo using a recombinant herpes simplex virus. Neuroscience 79:435-447.

Okoshi R, Ozaki T, Yamamoto H, Ando K, Koida N, Ono S, Koda T, Kamijo T, Nakagawara A, Kizaki H (2008) Activation of AMP-activated protein kinase induces p53-dependent apoptotic cell death in response to energetic stress. J Biol Chem 283:3979-3987.

Onken B, Driscoll M (2010) Metformin induces a dietary restriction-like state and the oxidative stress response to extend $C$. elegans healthspan via AMPK, LKB1, and SKN-1. PLoS One 5:e8758.

Patel BP, Safdar A, Raha S, Tarnopolsky MA, Hamadeh MJ (2010) Caloric restriction shortens lifespan through an increase in lipid peroxidation, inflammation and apoptosis in the G93A mouse, an animal model of ALS. PLoS One 5:e9386.

Pedrini S, Sau D, Guareschi S, Bogush M, Brown RH Jr, Naniche N, Kia A, Trotti D, Pasinelli P (2010) ALS-linked mutant SOD1 damages mitochondria by promoting conformational changes in bcl-2. Hum Mol Genet 19:2974-2986.

Puigserver P, Spiegelman BM (2003) Peroxisome proliferator-activated receptor-gamma coactivator 1 alpha (PGC-1 alpha): Transcriptional coactivator and metabolic regulator. Endocr Rev 24:78-90.

Rothstein JD (2009) Current hypotheses for the underlying biology of amyotrophic lateral sclerosis. Ann Neurol 65 [Suppl 1]:S3-S9.

Shakkottai VG, do Carmo Costa M, Dell'Orco JM, Sankaranarayanan A, Wulff H, Paulson HL (2011) Early changes in cerebellar physiology accompany motor dysfunction in the polyglutamine disease spinocerebellar ataxia type 3. J Neurosci 31:13002-13014.

Shan X, Chiang PM, Price DL, Wong PC (2010) Altered distributions of gemini of coiled bodies and mitochondria in motor neurons of TDP-43 transgenic mice. Proc Natl Acad Sci U S A 107:16325-16330.

Shih JD, Hunter CP (2011) SID-1 is a dsRNA-selective dsRNA-gated channel. RNA 17:1057-1065.

Shin JH, Ko HS, Kang H, Lee Y, Lee YI, Pletinkova O, Troconso JC, Dawson VL, Dawson TM (2011) PARIS (ZNF746) repression of PGC-1alpha contributes to neurodegeneration in Parkinson's disease. Cell 144:689-702.

Soukas AA, Kane EA, Carr CE, Melo JA, Ruvkun G (2009) Rictor/TORC2 regulates fat metabolism, feeding, growth, and life span in Caenorhabditis elegans. Genes Dev 23:496-511.

Spampanato J, Gu X, Yang XW, Mody I (2008) Progressive synaptic pathology of motor cortical neurons in a BAC transgenic mouse model of Huntington's disease. Neuroscience 157:606-620.

Timmons L, Fire A (1998) Specific interference by ingested dsRNA. Nature 395:854

Turnley AM, Stapleton D, Mann RJ, Witters LA, Kemp BE, Bartlett PF (1999) Cellular distribution and developmental expression of AMPactivated protein kinase isoforms in mouse central nervous system. J Neurochem 72:1707-1716

Viollet B, Andreelli F, Jorgensen SB, Perrin C, Geloen A, Flamez D, Mu J, Lenzner C, Baud O, Bennoun M, Gomas E, Nicolas G, Wojtaszewski JF, Kahn A, Carling D, Schuit FC, Birnbaum MJ, Richter EA, Burcelin R, Vaulont S (2003) The AMP-activated protein kinase alpha2 catalytic subunit controls whole-body insulin sensitivity. J Clin Invest 111:91-98.

Wang J, Farr GW, Hall DH, Li F, Furtak K, Dreier L, Horwich AL (2009) An ALS-linked mutant SOD1 produces a locomotor defect associated with aggregation and synaptic dysfunction when expressed in neurons of Caenorhabditis elegans. PLoS Genet 5:e1000350.

Weydt P, Pineda VV, Torrence AE, Libby RT, Satterfield TF, Lazarowski ER, Gilbert ML, Morton GJ, Bammler TK, Strand AD, Cui L, Beyer RP, Easley 
CN, Smith AC, Krainc D, Luquet S, Sweet IR, Schwartz MW, La Spada AR (2006) Thermoregulatory and metabolic defects in Huntington's disease transgenic mice implicate PGC-1alpha in Huntington's disease neurodegeneration. Cell Metab 4:349-362.

Weydt P, Soyal SM, Gellera C, Didonato S, Weidinger C, Oberkofler H, Landwehrmeyer GB, Patsch W (2009) The gene coding for PGC-1alpha modifies age at onset in Huntington's disease. Mol Neurodegener 4:3-9.

Winston WM, Molodowitch C, Hunter CP (2002) Systemic RNAi in C. elegans requires the putative transmembrane protein SID-1. Science 295:2456-2459.

Xu YF, Gendron TF, Zhang YJ, Lin WL, D'Alton S, Sheng H, Casey MC, Tong J, Knight J, Yu X, Rademakers R, Boylan K, Hutton M, McGowan E,
Dickson DW, Lewis J, Petrucelli L (2010) Wild-type human TDP-43 expression causes TDP-43 phosphorylation, mitochondrial aggregation, motor deficits, and early mortality in transgenic mice. J Neurosci 30:10851-10859.

Zhao Z, Lange DJ, Voustianiouk A, MacGrogan D, Ho L, Suh J, Humala N, Thiyagarajan M, Wang J, Pasinetti GM (2006) A ketogenic diet as a potential novel therapeutic intervention in amyotrophic lateral sclerosis. BMC Neurosci 7:29-39.

Zhou J, Yi J, Fu R, Liu E, Siddique T, Rios E, Deng HX (2010) Hyperactive intracellular calcium signaling associated with localized mitochondrial defects in skeletal muscle of an animal model of amyotrophic lateral sclerosis. J Biol Chem 285:705-712. 